\title{
Toda Lattice Hierarchy and Conservation Laws
}

\author{
Takashi Takebe
}

Department of Mathematics, Faculty of Science, University of Tokyo, Hongo 7-3-1 Bunkyo-ku, Tokyo 113, Japan

\begin{abstract}
Time evolutions of the Toda lattice hierarchies of Ueno and Takasaki are induced by Hamiltonians which are conservation laws for the original (one and two dimensional) Toda lattice obtained by Olive and Turok. Moreover these Hamiltonians for two dimensional Toda lattice hierarchy are also conserved quantities of the two component KP hierarchy in which that system is embedded. The one dimensional Toda lattice hierarchy is characterized by the bilinear relations, and a new version of the one dimensional Toda lattice hierarchy is constructed. Generalized Toda lattice hierarchies associated to all affine Lie algebras are presented.
\end{abstract}

\section{Introduction}

The Toda lattice has been, together with the Kortweg-de Vries (KdV) equation, one of the most important completely integrable non-linear systems, many features of which have been revealed by various methods. In this paper we will investigate the interrelationship between the Toda lattice hierarchy (K. Ueno, K. Takasaki [U-T]) and the structure of the Toda lattice as a Hamiltonian system with infinitely many constants of the motion (D. Olive, N. Turok [O-T1,2,3]), and conserved quantities of the multi component KP hierarchy. We also give a characterization by the bilinear relations and another version of one dimensional Toda lattice hierarchy, and present a candidate for the generalized Toda lattice hierarchy associated to any affine Lie algebra.

Ueno and Takasaki [U-T] introduced the Toda lattice hierarchy (hereafter we will abbreviate it to TL hierarchy), inspired by the theory of the KadomtsevPetviashvili (KP) hierarchies, and investigated its Lax representation, ZakharovShabat representation, the linearization, the $\tau$ function and its bilinear equations of Hirota-type etc., and showed that the TL hierarchy is embedded into the 2 component KP hierarchy. They also defined the periodic reduction and the restriction of the system to the one dimensional sector.

On the other hand, Olive and Turok [O-T1,2,3] made full use of the classical $r$ matrix (they call it the $\mathbf{P}$ operator; cf. $[\mathrm{Fa}, \mathrm{Fa}-\mathrm{T}]$ ) introduced from the theory 
of the quantum inverse scattering method to get infinitely many constants of the motion for the one and two dimensional Toda lattice. Their fundamental principle is, first, to express the simultaneous commutation relation using the $r$ matrix, and then, to construct infinitely many conserved quantities, and finally, regarding them as Hamiltonians, to consider time evolutions corresponding to them, which commute with each other. The final result gets the form of 0 -curvature conditions.

The main theorem of this paper is the following:

\section{Theorem}

(i) Time evolutions of the 1 dimensional TL hierarchy are induced as a Hamiltonian system by the conserved quantities of the original Toda lattice obtained in [O-T1]. (ii) Time evolutions of periodic reduced TL hierarchy are induced as a Hamiltonian system by the conserved quantities of the $A_{l-1}^{(1)}$ type 2 dimensional Toda lattice obtained in [O-T2,3].

(iii) These Hamiltonians of the TL hierarchy are conserved quantities of the 2 component KP hierarchy in which the TL hierarchy is embedded.

Here in the above theorem for the 1 dimensional case, some physical justification is needed, for the theory developed in $[\mathrm{O}-\mathrm{T} 1,2,3]$ is based on the finite rank Kac-Moody Lie algebras, while the TL hierarchy consists of $\mathbf{Z} \times \mathbf{Z}$ matrix of formal power series. To avoid handling divergences, we construct a new hierarchy by characterizing the Lax pairs algebraically which we get, following the prescription in [O-T1], from the formal Hamiltonians. Then we show that this new hierarchy is nothing but the one dimensional TL hierarchy of [U-T].

For the two dimensional system, we define a new system which naturally includes the system of $[\mathrm{O}-\mathrm{T} 2,3]$ and corresponds to an affine $\mathrm{Kac}-\mathrm{Moody}$ algebra. This system associated to $A_{l-1}^{(1)}$ type Lie algebra is identical with the $l$-periodic TL hierarchy, when it is represented as infinite matrices. (M. Fukuma advised the author to deal with the finite periodic lattice rather than the infinite lattice.)

While Olive and Turok discuss along the formulation of the inverse scattering method convenient for quantization (cf. $[\mathrm{Fa}],[\mathrm{Fa}-\mathrm{T}]$ ), in $[\mathrm{R}-\mathrm{S}]$ the "half" of the TL hierarchy is constructed by a clever use of the co-adjoint orbit method and the central extension of Lie algebras.

The present paper is organized as follows:

In Sect. 1.1, we recall the materials in [U-T] which are needed later, without fixing gauge specified by a parameter $\alpha$; [U-T] used $\alpha=1 / 2$ gauge but $\alpha=0$ gauge is of great importance and, at the same time, interesting from the historical point of view. (This gauge brings historic Lax operators for the Toda lattice in sight of the theory of the TL hierarchy.) Then we discuss the one dimensional TL hierarchy and, when the gauge is fixed appropriately $(\alpha=0)$, the system has high symmetry, which allows the characterization of the one dimensional TL hierarchy by the bilinear relations of the wave matrices.

In Sect. 2, making use of the classical $r$ matrix as in [O-T1], we construct infinitely many Lax pairs for the one dimensional Toda lattice and investigate its structure as a hierarchy of Sato-type. On the other hand this system has a natural interpretation as a Hamiltonian system, which we will explain in Sect. 2.1. From 
the bilinear relations for the wave matrices, we know that this " $r$ matrix type" one dimensional TL hierarchy is identified with that of $[\mathrm{U}-\mathrm{T}]$ through a simple linear transformation of time variables.

In Sect. 3, first we recall the theory of Olive and Turok [O-T2,3], generalizing it to all affine Lie algebras including twisted ones. In this theory the classical $r$ matrix plays an essential role, especially in the formula "fundamental Poisson relation." Then, through the zero curvature conditions obtained, we can introduce non-local elements of gauge groups, which behave just like solutions of the linearized problem of the TL hierarchy (wave matrices). With the help of this "wave matrix," the similarity between the theory of $[\mathrm{O}-\mathrm{T} 2,3]$ and the TL hierarchy becomes clear, and thus a candidate for the generalized TL hierarchy associated to an arbitrary affine Lie algebra is presented. As mentioned above, this system is identified with the $l$-reduced TL hierarchy in the $A_{l-1}^{(1)}$ case.

In Sect. 4, changing the standing point, we construct these conserved quantities quite algebraically. [U-T] has proved that the TL hierarchy is embedded in 2 component KP hierarchy. Therefore it is natural to ask how the conserved quantities are embedded in 2 component KP hierarchy. Here, after recalling the $r$ component KP hierarchy, we calculate its conserved quantities from the solution of the linear problem (the wave operator) under suitable boundary conditions. This is a generalization of the results of $[\mathrm{W}, \mathrm{Ch} 1, \mathrm{Fl}$ and $\mathrm{Sa}]$ to the matrix case. Comparing their expressions in $\tau$ function, we know that the Hamiltonians of the TL hierarchy and the conserved quantities of the 2 component KP hierarchy coincide. The real reason of this coincidence has not yet been explained and should be further studied.

We also remark that TL hierarchy and KP hierarchy are deeply related to the 2 dimensional field theories in physics, and the conserved quantities obtained in the present paper are important in the theory of deformations of conformal field theories [Fu-T].

\section{Notations}

- Usual abbreviations for differentiations: e.g.

- Let $(a(s))_{s \in \mathbf{Z}}$ be a series, then:

$$
\partial_{x}=\frac{\partial}{\partial x}
$$

$$
\operatorname{diag}[a(s)]:=\left(a(i) \delta_{i j}\right)_{i, j \in \mathbf{Z}},
$$

i.e., the diagonal matrix, diagonal elements of which are $a(s)$ 's.

- $\Lambda^{ \pm 1}:=\left(\delta_{i \pm 1 j}\right)_{l, j \in \mathbf{Z}}$ : the shift matrices.

Hence $\mathbf{Z} \times \mathbf{Z}$ matrix is written in a convenient form as

$$
\left(\begin{array}{ccccc}
\ddots & \ddots & \ddots & & \\
\ddots & a_{0}(-1) & a_{1}(-1) & a_{2}(-1) & \\
\ddots & a_{-1}(0) & a_{0}(0) & a_{1}(0) & \ddots \\
& a_{-2}(1) & a_{-1}(1) & a_{0}(1) & \ddots \\
& & \ddots & \ddots & \ddots
\end{array}\right)=\sum_{j \in Z} \operatorname{diag}\left[a_{j}(s)\right] \Lambda^{j} .
$$


- For $l \in N \mathbf{Z} \times \mathbf{Z}$ matrix $A=\left(a_{i j}\right)$ is called $l$ periodic if for all $i, j \in \mathbf{Z} a_{i j}=a_{i+l, j+l}$. - For a matrix $A=\left(a_{i j}\right)$, matrices $A_{+}, A_{-}, A_{0}$ are defined by:

$$
\begin{aligned}
& \left(A_{+}\right)_{i j}= \begin{cases}a_{i j}, & \text { if } i<j, \\
0, & \text { otherwise; }\end{cases} \\
& \left(A_{-}\right)_{i j}= \begin{cases}a_{i j}, & \text { if } i>j, \\
0, & \text { otherwise; }\end{cases} \\
& \left(A_{0}\right)_{i j}= \begin{cases}a_{i j}, & \text { if } i=j, \\
0, & \text { otherwise. }\end{cases}
\end{aligned}
$$

Hence, $A=A_{+}+A_{0}+A_{-}$. Our usage of ${ }_{+}$is different from that in [U-T].

- Hereafter, "function" means a formal power series.

\section{Toda Lattice Hierarchy}

1. Reviews of the TL Hierarchy of Ueno and Takasaki. Here we recall the Toda lattice hierarchy (TL hierarchy) [U-T] in a slightly modified form, i.e., with one parameter $\alpha$ which designates the gauge.

Let $L^{ \pm}, B_{n}$ be the following $\mathbf{Z} \times \mathbf{Z}$ matrix valued functions of $x_{ \pm}=\left(x_{ \pm 1}, x_{ \pm 2}, \ldots\right)$ :

$$
L_{+}=\sum_{-\infty<j \leqq 1} \operatorname{diag}\left[b_{j}^{+}(s)\right] \Lambda^{j}, \quad L_{-}=\sum_{-1 \leqq j<\infty} \operatorname{diag}\left[b_{j}^{-}(s)\right] \Lambda^{j},
$$

with

$$
\begin{aligned}
b_{1}^{+}(s)^{1 / 2+\alpha} & =b_{-1}^{-}(s+1)^{-1 / 2+\alpha}, \quad b_{ \pm 1}^{ \pm} \neq 0, \\
B_{n} & =\left(L_{+}^{n}\right)_{+}+\left(\frac{1}{2}+\alpha\right)\left(L_{+}^{n}\right)_{0}, \\
B_{-n} & =\left(L_{-}^{n}\right)_{-}+\left(\frac{1}{2}-\alpha\right)\left(L_{-}^{n}\right)_{0}, \quad n=1,2, \ldots,
\end{aligned}
$$

where $b_{j}^{ \pm}(s)=b_{j}^{ \pm}(s ; x)$ are functions of $x_{+}$and $x_{-}$.

The Toda lattice hierarchy under $\alpha$-gauge is, by definition, the compatibility conditions for the linear problem,

$$
\begin{array}{rlrl}
L_{+} W^{(\infty)} & =W^{(\infty)} \Lambda, & L_{-} W^{(0)}=W^{(0)} \Lambda^{-1} \\
\partial_{x_{n}} W & =B_{n} W, & & n= \pm 1, \pm 2, \ldots,
\end{array}
$$

of the $\mathbf{Z} \times \mathbf{Z}$ matrix valued unknown functions $W=W^{(\infty)}$ and $W^{(0)}$.

Explicitly, the TL hierarchy is the following system for the unknown functions $b_{j}^{ \pm}$; for all $m, n= \pm 1, \pm 2, \ldots$,

$$
\begin{aligned}
\partial_{x_{n}} L_{ \pm} & =\left[B_{n}, L^{ \pm}\right], \\
{\left[\partial_{x_{n}}-B_{n}, \partial_{x_{m}}-B_{m}\right] } & =\partial_{x_{m}} B_{n}-\partial_{x_{n}} B_{m}+\left[B_{n}, B_{m}\right]=0,
\end{aligned}
$$

Proposition 1.1. Under the condition (1.1), (1.3L) and (1.3ZS) are equivalent to each other.

Proof. We prove that $(1.3 \mathrm{~L})$ implies $(1.3 \mathrm{ZS})$. The converse can be proved in the same way as Theorem 1.1 of [U-T]. Let us introduce 1 -forms $\omega, \Omega_{ \pm}, \xi, X_{ \pm}^{(\infty)}$ by 


$$
\begin{aligned}
\omega & :=\sum_{m=1}^{\infty} L_{+}^{m} d x_{m}, & \xi & :=\sum_{m=1}^{\infty} L_{-}^{m} d x_{-m}, \\
\Omega_{+} & :=(\omega)_{+}+\left(\frac{1}{2}+\alpha\right)(\omega)_{0}, & X_{+}^{(\infty)} & :=(\xi)_{+}+\left(\frac{1}{2}+\alpha\right)(\xi)_{0}, \\
\Omega_{-} & :=(\omega)_{-}+\left(\frac{1}{2}-\alpha\right)(\omega)_{0}, & X_{-}^{(\infty)} & :=(\xi)_{-}+\left(\frac{1}{2}-\alpha\right)(\xi)_{0} .
\end{aligned}
$$

We denote the exterior differentiations with respect to $x_{ \pm}$as $d_{ \pm}$. Then

$$
\begin{array}{ll}
d_{+} \omega=\omega \wedge \Omega_{+}+\Omega_{+} \wedge \omega, & d_{+} \xi=\xi \wedge \Omega_{+}+\Omega_{+} \wedge \xi, \\
d_{-} \omega=\omega \wedge X_{-}^{(\infty)}+X_{-}^{(\infty)} \wedge \omega, & d_{-} \xi=\xi \wedge X_{-}^{(\infty)}+X_{-}^{(\infty)} \wedge \xi,
\end{array}
$$

follow from (1.3L). Since $\omega=\Omega_{+}+\Omega_{-}$and $\omega \wedge \omega=0$, the first equation of (1.4) yields

$$
d_{+} \omega=\Omega_{+} \wedge \Omega_{+}-\Omega_{-} \wedge \Omega_{-} .
$$

As $B_{n}$ is upper triangular matrix for $n>0$,

$$
\Omega_{+} \wedge \Omega_{+}=\sum_{0<n<m}\left[B_{n}, B_{m}\right] d x_{n} \wedge d x_{m}
$$

is strictly upper triangular. Similarly $\Omega_{-} \wedge \Omega_{-}$is strictly lower triangular. Hence

$$
d_{+} \Omega_{+}=\Omega_{+} \wedge \Omega_{+},
$$

which is (1.3ZS) $n, m>0$. The last equation of (1.4) yields (1.3ZS) $n, m<0$ likewise.

Using the second and third equation of (1.4), we obtain

$$
\begin{aligned}
d_{-} & \Omega_{+}+d_{+} X_{-}^{(\infty)}-\left(X_{-}^{(\infty)} \wedge \Omega_{+}+\Omega_{+} \wedge X_{-}^{(\infty)}\right) \\
& =-d_{-} \Omega_{-}+d_{+} X_{-}^{(\infty)}+\left(X_{-}^{(\infty)} \wedge \Omega_{-}+\Omega_{-} \wedge X_{-}^{(\infty)}\right) \\
& =d_{-} \Omega_{+}-d_{-} X_{+}^{(\infty)}+\left(X_{+}^{(\infty)} \wedge \Omega_{+}+\Omega_{+} \wedge X_{+}^{(\infty)}\right) .
\end{aligned}
$$

The left-hand side of this equation is upper and lower triangular at the same time, that is, diagonal. The diagonal part is

$$
\begin{aligned}
& \left(-d_{-} \Omega_{-}+d_{+} X_{-}^{(\infty)}\right)_{0}=\left(\frac{1}{2}-\alpha\right)\left(-d_{-} \omega+d_{+} \xi\right)_{0} \\
& =\left(d_{-} \Omega_{+}-d_{+} X_{+}^{(\infty)}\right)_{0}=-\left(\frac{1}{2}+\alpha\right)\left(-d_{-} \omega+d_{+} \xi\right)_{0}=0 .
\end{aligned}
$$

This proves (1.3ZS) in the case when $n$ and $m$ have opposite signs.

If $L_{ \pm}$are solutions of the TL hierarchy, then there exist solution matrices (wave matrices) $W^{\left(\begin{array}{c}0 \\ \infty\end{array}\right)}\left(=W^{(0)}\right.$ or $\left.W^{(\infty)}\right)$ to the linear problem (1.2) such that

$$
\begin{aligned}
W^{(\infty)}(x) & =\hat{W}^{(\infty)}(x) \exp \xi\left(x_{+}, \Lambda\right), \\
W^{(0)}(x) & =\hat{W}^{(0)}(x) \exp \xi\left(x_{-}, \Lambda^{-1}\right),
\end{aligned}
$$

where

$$
\hat{W}^{\left(\begin{array}{c}
0 \\
\infty
\end{array}\right)}(x)=\sum_{j=0}^{\infty} \operatorname{diag}\left[\hat{w}_{j}^{(0)}(s ; x)\right] \Lambda^{ \pm j}
$$

with

$$
\hat{w}_{0}^{(0)}(s ; x)^{-1 / 2+\alpha}=\hat{w}_{0}^{(\infty)}(s ; x)^{1 / 2+\alpha}, \quad \hat{w}_{0}^{(0)} \neq 0
$$


and $\xi\left(x_{ \pm}, \Lambda^{ \pm 1}\right)=\sum_{n=1}^{\infty} x_{ \pm n} \Lambda^{ \pm n}$. The proof is similar to that of Theorem 1.2 of [U-T]

The following proposition gives the complete characterization of solutions of the TL hierarchy in terms of these wave matrices.

\section{Proposition 1.2.}

(i) Wave matrices satisfy the following bilinear relations for all $x, x^{\prime}$;

$$
\mathbf{T}:=W^{(\infty)}(x) W^{(\infty)}\left(x^{\prime}\right)^{-1}=W^{(0)}(x) W^{(0)}\left(x^{\prime}\right)^{-1} .
$$

$\mathbf{T}$ is called the transition matrix from $x$ to $x^{\prime}$.

(ii) Conversely, if $W^{\left(\begin{array}{c}0 \\ \infty\end{array}\right)}$ of the form (1.5) satisfy (1.6), then one can set

$$
\begin{aligned}
& L_{+}:=W^{(\infty)} \Lambda W^{(\infty)-1}\left(=\hat{W}^{(\infty)} \Lambda \hat{W}^{(\infty)-1}\right), \\
& L_{-}:=W^{(0)} \Lambda^{-1} W^{(0)-1}\left(=\hat{W}^{(0)} \Lambda^{-1} \hat{W}^{(0)-1}\right)
\end{aligned}
$$

to obtain the solutions of (1.2) and (1.3).

(The products of infinite matrices above (e.g. in (1.5), (1.6)) are well-defined and convergent when the topology of the ring of formal power series is introduced by degree: $\operatorname{deg} x_{n}=|n|$. In what follows, we don't make this kind of remarks.)

Proof. It is easy and differs from that of Theorem 1.5, [U-T] only in the point that one must pay attention to the diagonal part of wave matrices.

(i) is also a direct consequence of the uniqueness of the solution of the Cauchy problem:

$$
\begin{aligned}
\frac{\partial}{\partial x_{n}} \mathbf{T}\left(x, x^{\prime}\right) & =B_{n}(x) \mathbf{T}\left(x, x^{\prime}\right), \\
\frac{\partial}{\partial x_{n}^{\prime}} \mathbf{T}\left(x, x^{\prime}\right) & =-\mathbf{T}\left(x, x^{\prime}\right) B_{n}\left(x^{\prime}\right), \\
\mathbf{T}(x, x) & =1 .
\end{aligned}
$$

The relation (1.6) is formally equivalent to

$$
W^{(0)}(x)^{-1} W^{(\infty)}(x)=A=\text { coistant matrix. }
$$

This gives the Riemann-Hilbert decomposition of an element of $G L(\infty)$.

We define an important function $u(s ; x)$ such that

$$
\hat{w}_{0}^{(0)}(s ; x)=e^{(1 / 2+\alpha) u(s)}, \quad \hat{w}_{0}^{(\infty)}(s ; x)=e^{(-1 / 2+\alpha) u(s)} .
$$

Then one of the equations (1.3ZS) $n=1, m=-1:\left[\partial_{x_{1}}-B_{1}, \partial_{x_{-1}}-B_{-1}\right]=0$ is nothing but the original 2-dimensional Toda lattice equation

$$
\partial_{x_{1}} \partial_{x_{-1}} u(s ; x)=e^{u(s)-u(s-1)}-e^{u(s+1)-u(s)} .
$$

Proposition 1.3. Let $L_{ \pm}^{(\alpha)}$ be solutions of the TL hierarchy of $\alpha$-gauge, and define $B_{n}^{(\alpha)}=B_{n}$ as above and

$$
g=g_{\alpha \beta}:=\operatorname{diag}\left[e^{(\alpha-\beta) u(s)}\right]
$$


Then

$$
L_{ \pm}^{(\beta)}:=g^{-1} L_{ \pm}^{(\alpha)} g
$$

is a solution of the TL hierarchy of $\beta$-gauge, and

$$
\begin{aligned}
B_{n}^{(\beta)} & =g^{-1} B_{n}^{(\alpha)} g+\partial_{x_{n}} g^{-1} g, \\
W^{(\beta)} & =g^{-1} W^{(\alpha)}, \quad \hat{W}^{(\beta)}=g^{-1} \hat{W}^{(\alpha)} .
\end{aligned}
$$

Proof. It is straightforward, using Proposition 1.2.

This proposition states the gauge invariance of solutions of the TL hierarchy, and allows us to use all the results of [U-T] which treats the TL hierarchy of $\alpha=1 / 2$ gauge. Here we recall some of them without fixing gauge. (Later we will use mainly $\alpha=0$ gauge.)

For $W^{\left(\begin{array}{l}0 \\ \infty\end{array}\right)}$ above there exists the so-called $\tau$ function $\tau(s ; x)$ such that

$$
\begin{aligned}
\hat{W}^{(\infty)}(x) & =\sum_{j=0}^{\infty} \operatorname{diag}\left[\frac{p_{j}\left(-\tilde{\partial}_{x}\right) \tau(s)}{\tau(s+1)^{1 / 2-\alpha} \tau(s)^{1 / 2+\alpha}}\right] \Lambda^{-j}, \\
\hat{W}^{(0)}(x) & =\sum_{j=0}^{\infty} \operatorname{diag}\left[\frac{p_{j}\left(-\tilde{\partial}_{y}\right) \tau(s+1)}{\tau(s+1)^{1 / 2-\alpha} \tau(s)^{1 / 2+\alpha}}\right] \Lambda^{j}, \\
\hat{W}^{(\infty)}(x)^{-1} & =\sum_{j=0}^{\infty} \Lambda^{-j} \operatorname{diag}\left[\frac{p_{j}\left(\tilde{\partial}_{x}\right) \tau(s+1)}{\tau(s+1)^{1 / 2+\alpha} \tau(s)^{1 / 2-\alpha}}\right], \\
\hat{W}^{(0)}(x)^{-1} & =\sum_{j=0}^{\infty} \Lambda^{j} \operatorname{diag}\left[\frac{p_{j}\left(\tilde{\partial}_{y}\right) \tau(s)}{\tau(s+1)^{1 / 2+\alpha} \tau(s)^{1 / 2-\alpha}}\right],
\end{aligned}
$$

and for $n>0$

$$
\begin{aligned}
B_{n}= & \sum_{m=1}^{n} \operatorname{diag}\left[\frac{p_{n-m}\left(\tilde{D}_{x_{+}}\right) \tau(s+m+1) \cdot \tau(s)}{\tau(s+m+1)^{1 / 2+\alpha} \tau(s+m)^{1 / 2-\alpha} \tau(s+1)^{1 / 2-\alpha} \tau(s)^{1 / 2+\alpha}}\right] \Lambda^{m} \\
& +\left(\frac{1}{2}+\alpha\right) \operatorname{diag}\left[\frac{p_{n}\left(\tilde{D}_{x_{+}}\right) \tau(s+1) \cdot \tau(s)}{\tau(s+1) \tau(s)}\right] \\
B_{-n}= & \sum_{m=-n}^{-1} \operatorname{diag}\left[\frac{p_{n+m}\left(\tilde{D}_{x_{-}}\right) \tau(s+m) \cdot \tau(s+1)}{\tau(s+m+1)^{1 / 2+\alpha} \tau(s+m)^{1 / 2-\alpha} \tau(s+1)^{1 / 2-\alpha} \tau(s)^{1 / 2+\alpha}}\right] \Lambda^{m} \\
& +\left(\frac{1}{2}-\alpha\right) \operatorname{diag}\left[\frac{p_{n}\left(\tilde{D}_{x_{-}}\right) \tau(s) \cdot \tau(s+1)}{\tau(s+1) \tau(s)}\right]
\end{aligned}
$$

Here we use the notations

$$
\begin{aligned}
e^{\xi\left(x_{ \pm}, \lambda\right)} & =\sum_{j=0}^{\infty} p_{j}\left(x_{ \pm}\right) \lambda^{j}, \\
\tilde{\partial}_{x_{ \pm}} & =\left(\partial_{x_{ \pm}}, \frac{1}{2} \partial_{x_{ \pm}}, \frac{1}{3} \partial_{x_{ \pm}}, \ldots\right) .
\end{aligned}
$$

The symbol $D$ denotes Hirota's $D$-operator which is defined for a linear differential operator $F\left(\partial_{x}\right)$ by

$$
F\left(D_{x}\right) f(x) \cdot g(x)=\left.F\left(\partial_{x^{\prime}}\right) f\left(x+x^{\prime}\right) g\left(x-x^{\prime}\right)\right|_{x^{\prime}=0} .
$$


$u=u(s ; x)$ is expressed in terms of the $\tau$ function as

$$
e^{u(s)}=\frac{\tau(s+1)}{\tau(s)} .
$$

The following modified wave matrices and the modified $\tau$ function are also important:

$$
\begin{aligned}
\hat{V}^{(\infty)}(x) & =\hat{W}^{(\infty)}(x) \exp \left(-\xi\left(x_{-}, \Lambda^{-1}\right)\right), \\
\hat{V}^{(0)}(x) & =\hat{W}^{(0)}(x) \exp \left(-\xi\left(x_{+}, \Lambda\right)\right) \\
\tau^{\prime}(x) & =\tau(x) \exp \left(\sum_{n=1}^{\infty} n x_{n} x_{-n}\right) .
\end{aligned}
$$

Note that (1.8) and (1.9) also hold if $\hat{W}$ and $\tau$ are replaced to $\hat{V}$ and $\tau^{\prime}$ respectively, and that the $\tau$ (or $\tau^{\prime}$ ) function is uniquely determined up to arbitrariness of the factor $a^{s} b \exp \left(\sum_{n=1}^{\infty}\left(c_{n} x_{n}+d_{n} y_{n}\right)\right)$.

l-periodic reduction (or $A_{l-1}^{(1)}$ reduction) of the TL hierarchy is defined by imposing the following constraint on the TL hierarchy:

$$
L_{+}^{l}=\Lambda^{l}, \quad L_{-}^{l}=\Lambda^{-l} \text {. }
$$

Then $L_{ \pm}, B_{n}, \hat{W}^{\left(\begin{array}{c}0 \\ \infty\end{array}\right)}$ are $l$ periodic matrices (cf. Notations), and

$$
\partial_{x_{n}} L_{+}=\partial_{x_{n}} L_{-}=0
$$

for $n \equiv 0(\bmod l)$. We can also take the $\tau$ function as periodic in $s$ and independent of the variables $x_{n}(n \equiv 0(\bmod l))$ :

$$
\begin{aligned}
\tau^{\prime}(s ; x) & =\tau^{\prime}(s+l ; x), \\
\partial_{x_{n}} \tau^{\prime}(s ; x) & =\partial_{x_{n}} \tau^{\prime}(s ; x)=0, \quad n \equiv 0(\bmod l) .
\end{aligned}
$$

Since $u(s)=\log \left(\tau^{\prime}(s+1) / \tau^{\prime}(s)\right)$, this implies

$$
u(s)=u(s+l), \quad \sum_{s=0}^{l-1} u(s)=0 .
$$

As $u(s)$ is $l$-periodic, $g=g_{\alpha \beta}$ is also periodic;

$$
\left[g, \Lambda^{l}\right]=0 .
$$

This equation together with Proposition 1.3 means the gauge invariance of the constraint (1.10).

2. The 1-Dimensional TL Hierarchy. In this section we will transform the gauge of the TL hierarchy and discuss the 1-dimensional TL hierarchy reduced from the original one by freezing the evolution to the "space" direction.

First, regarding $x_{ \pm n}(n>0)$ as the light-cone variables, we introduce new space-time variables $z, t$ by

$$
z_{n}=\frac{1}{2}\left(x_{n}+x_{-n}\right), \quad t_{n}=\frac{1}{2}\left(x_{n}-x_{-n}\right), \quad n=1,2, \ldots .
$$


Then (1.2) is represented as

$$
\begin{aligned}
L_{+} W^{(\infty)} & =W^{(\infty)} \Lambda, \quad L_{-} W^{(0)}=W^{(0)} \Lambda^{-1}, \\
\partial_{z_{n}} W & =Q_{n} W \quad \text { with } \quad Q_{n}=B_{n}+B_{-n}, \\
\partial_{t_{n}} W & =X_{n} W \quad \text { with } \quad X_{n}=B_{n}-B_{-n} .
\end{aligned}
$$

For example, $Q_{1}$ and $X_{1}$ are of the well known form as follows: (cf. [M-O-P])

$$
\begin{aligned}
& Q_{1}=\left(\begin{array}{ccccc}
\ddots & \ddots & & \\
\ddots & \frac{1}{2} \partial_{t_{1}} u(-1) & e^{(u(0)-u(-1)) / 2} & & \\
& e^{(u(0)-u(-1)) / 2} & \frac{1}{2} \partial_{t_{1}} u(0) & e^{(u(1)-u(0)) / 2} & \\
& & e^{(u(1)-u(0)) / 2} & \frac{1}{2} \partial_{t_{1}} u(1) & \ddots \\
0 & & \ddots & \ddots
\end{array}\right), \\
& X_{1}=\left(\begin{array}{ccccc}
\ddots & \ddots & & & \\
\ddots & \frac{1}{2} \partial_{z_{1}} u(-1) & e^{(u(0)-u(-1)) / 2} & & \\
& -e^{(u(0)-u(-1)) / 2} & \frac{1}{2} \partial_{z_{1}} u(0) & e^{(u(1)-u(0)) / 2} & \\
0 & & -e^{(u(1)-u(0)) / 2} & \frac{1}{2} \partial_{z_{1}} u(1) & \ddots \\
0 & & & \ddots & \ddots
\end{array}\right) .
\end{aligned}
$$

The 1-dimensional TL hierarchy is defined as the TL hierarchy with the additional gauge invariant constraint

$$
L_{+}+L_{+}^{-1}=L_{-}+L_{-}^{-1}
$$

or equivalently

$$
\partial_{z_{n}} \hat{V}=0, \text { for all } n=1,2, \ldots
$$

or

$$
\tau^{\prime} \text { can be chosen so that } \partial_{z_{n}} \tau^{\prime}=0 \text { for all } n \text {. }
$$

(cf. [U-T]; note the arbitrariness of the $\tau$ function mentioned in Sect. 1.1.)

Hence if we set

$$
\begin{aligned}
V^{\left(\begin{array}{l}
0 \\
\infty
\end{array}\right)}(t) & :=\hat{V}^{\left(\begin{array}{c}
0 \\
\infty_{\infty}
\end{array}\right)}(t) \exp \left(\xi(t, \Lambda)-\xi\left(t, \Lambda^{-1}\right)\right) \\
& =W^{\left(\begin{array}{c}
0 \\
\infty_{0}
\end{array}\right)}(z, t) \exp \left(-\xi(z, \Lambda)-\xi\left(z, \Lambda^{-1}\right)\right),
\end{aligned}
$$

we get the following proposition by the same method as Proposition 1.17 of [U-T].

\section{Proposition 1.4.}

(i) If $L_{ \pm}, Q_{n}$ and $X_{n}$ solve the 1-dimensional TL hierarchy, then they depend on only $t$.

(ii) Under the same assumption as above, $V(t)$ solves the linear problem

$$
\begin{aligned}
& Q_{1} V(t)=V(t)\left(\Lambda+\Lambda^{-1}\right), \\
& \partial_{t_{n}} V(t)=X_{n} V(t), \quad n=1,2, \ldots .
\end{aligned}
$$

The compatibility condition of this system amounts to the Lax representation

$$
\partial_{t_{n}} Q_{1}=\left[X_{n}, Q_{1}\right], \quad n=1,2, \ldots
$$


Especially $\partial_{t_{1}} Q_{1}=\left[X_{1}, Q_{1}\right]$ is the first Lax representation for the Toda lattice obtained by Flaschka.

Hereafter we fix the gauge $\alpha=0$. Then, using (1.8) and (1.9) with $\hat{V}$ and $\tau^{\prime}$, the following symmetry is easily seen.

\section{Proposition 1.5.}

For the 1-dimensional TL hierarchy

$$
\begin{aligned}
{ }^{t} Q_{n} & =Q_{n}, \\
{ }^{t} \hat{V}^{(\infty)} & =\left(\hat{V}^{(0)}\right)^{-1}, \\
{ }^{t} V^{(\infty)} & =\left(V^{(0)}\right)^{-1} .
\end{aligned}
$$

Moreover the bilinear relations characterizing the 1-dimensional TL hierarchy are expressed in terms of only $V$.

\section{Theorem 1.6.}

(i) For the 1-dimensional TL hierarchy,

$$
V^{(\infty)}(t) V^{(\infty)}\left(t^{\prime}\right)^{-1}=V^{(0)}(t) V^{(0)}\left(t^{\prime}\right)^{-1}
$$

holds for all $t$ and $t^{\prime}$.

(ii) Conversely, for an invertible lower triangular matrix $\hat{V}(t)$, suppose

and

$$
V^{(\infty)}(t):=\hat{V}(t) \exp \left(\xi(t, \Lambda)-\xi\left(t, \Lambda^{-1}\right)\right)
$$

satisfy

$$
V^{(0)}:={ }^{t} V^{(\infty)-1}
$$

$$
\begin{aligned}
V^{(\infty)}(t) V^{(\infty)}\left(t^{\prime}\right)^{-1} & =V^{(0)}(t) V^{(0)}\left(t^{\prime}\right)^{-1} \\
V^{(\infty)}(t)\left(\Lambda+\Lambda^{-1}\right) V^{(\infty)}(t)^{-1} & =V^{(0)}(t)\left(\Lambda+\Lambda^{-1}\right) V^{(0)}(t)^{-1}
\end{aligned}
$$

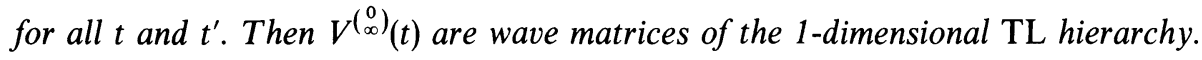
Proof.

(i) According to Proposition 1.2 and (1.13),

$$
\begin{aligned}
& V^{(\infty)}(t) \exp \left(\xi(z, \Lambda)+\xi\left(z, \Lambda^{-1}\right)-\xi\left(z^{\prime}, \Lambda\right)-\xi\left(z^{\prime}, \Lambda^{-1}\right)\right) V^{(\infty)}\left(t^{\prime}\right)^{-1} \\
& \quad=V^{(0)}(t) \exp \left(\xi(z, \Lambda)+\xi\left(z, \Lambda^{-1}\right)-\xi\left(z^{\prime}, \Lambda\right)-\xi\left(z^{\prime}, \Lambda^{-1}\right)\right) V^{(0)}\left(t^{\prime}\right)^{-1}
\end{aligned}
$$

Equation (1.13) and $\partial_{z_{n}} W=Q_{n} W$ imply

$$
V(t)\left(\Lambda^{n}+\Lambda^{-n}\right)=Q_{n} V(t) .
$$

Since $\xi(z, \Lambda)+\xi\left(z, \Lambda^{-1}\right)=\sum_{n=1}^{\infty} z_{n}\left(\Lambda^{n}+\Lambda^{-n}\right)$, applying the above formula to (1.17), we obtain

$$
\begin{aligned}
& \exp \left(\sum_{n=1}^{\infty} z_{n} Q_{n}(t)\right) V^{(\infty)}(t) V^{(\infty)}\left(t^{\prime}\right)^{-1} \exp \left(-\sum_{n=1}^{\infty} z_{n}^{\prime} Q_{n}\left(t^{\prime}\right)\right) \\
& \quad=\exp \left(\sum_{n=1}^{\infty} z_{n} Q_{n}(t)\right) V^{(0)}(t) V^{(0)}\left(t^{\prime}\right)^{-1} \exp \left(-\sum_{n=1}^{\infty} z_{n}^{\prime} Q_{n}\left(t^{\prime}\right)\right)
\end{aligned}
$$


Hence

$$
V^{(\infty)}(t) V^{(\infty)}\left(t^{\prime}\right)^{-1}=V^{(0)}(t) V^{(0)}\left(t^{\prime}\right)^{-1}
$$

(ii) First we set

$$
Q(t):=V^{(\infty)}(t)\left(\Lambda+\Lambda^{-1}\right) V^{(\infty)}(t)^{-1}
$$

Then

$$
\begin{aligned}
Q(t) & ={ }^{t} Q(t), \\
Q(t) V^{\left(\begin{array}{c}
0 \\
\infty_{0}
\end{array}\right)}(t) & =V^{\left({ }_{\infty}^{0}\right)}(t)\left(\Lambda+\Lambda^{-1}\right)
\end{aligned}
$$

follow from the second equation of (1.16). It is also shown by induction that there exists the polynomial $q_{n}$ of degree $n$ such that

$$
q_{n}(Q(t)) V^{\left(\begin{array}{c}
0 \\
\infty
\end{array}\right)}(t)=V^{\left(\begin{array}{c}
0 \\
\infty
\end{array}\right)}(t)\left(\Lambda^{n}+\Lambda^{-n}\right) \text {. }
$$

Now we show that

$$
\hat{W}^{(\infty)}:=\hat{V}(t) \exp \xi\left(y, \Lambda^{-1}\right), \quad \hat{W}^{(0)}:={ }^{t} \hat{V}(t)^{-1} \exp \xi(x, \Lambda)
$$

satisfy the condition in Proposition 1.2. Since

$$
W^{\left(\begin{array}{c}
0 \\
\infty
\end{array}\right)}=V^{\left(\begin{array}{c}
0 \\
\infty
\end{array}\right)}(t) \exp \sum z_{n}\left(\Lambda^{n}+\Lambda^{-n}\right)
$$

we have

$$
\begin{aligned}
& W^{(\infty)}(z, t) W^{(\infty)}\left(z^{\prime}, t^{\prime}\right)^{-1} \\
& \quad=V^{(\infty)}(t) \exp \left(\sum z_{n}\left(\Lambda^{n}+\Lambda^{-n}\right)-\sum z_{n}^{\prime}\left(\Lambda^{n}+\Lambda^{-n}\right)\right) V^{(\infty)}\left(t^{\prime}\right)^{-1} \\
& \stackrel{(1.18)}{=} \exp \left(\sum z_{n} q_{n}(Q(t))\right) V^{(\infty)}(t) V^{(\infty)}\left(t^{\prime}\right)^{-1} \exp \left(-\sum z_{n}^{\prime} q_{n}\left(Q\left(t^{\prime}\right)\right)\right) \\
& \stackrel{(1.16)}{=} \exp \left(\sum z_{n} q_{n}(Q(t))\right) V^{(0)}(t) V^{(0)}\left(t^{\prime}\right)^{-1} \exp \left(-\sum z_{n}^{\prime} q_{n}\left(Q\left(t^{\prime}\right)\right)\right) \\
& \stackrel{(1.18)}{=} W^{(0)}(z, t) W^{(0)}\left(z^{\prime}, t^{\prime}\right)^{-1} .
\end{aligned}
$$

Remark 1.7. We can formally write

$$
{ }^{t}\left(V^{(0)}(t)^{-1} V^{(\infty)}(t)\right)={ }^{t} V^{(\infty)}(t)^{t} V^{(0)}(t)^{-1}=V^{(0)}(t)^{-1} V^{(\infty)}(t) .
$$

Hence the 1-dimensional TL hierarchy corresponds to the Riemann-Hilbert decomposition of a symmetric matrix.

\section{The 1-Dimensional System and the Conservation Laws}

1. Classicalr Matrix and Lax Pairs. Here we recall about the relationship between Hamiltonian system and Lax pairs, making use of the classical $r$ matrix [O-T1], [Ko].

Suppose for a dynamical system the time evolution of the observable $f$ is determined in terms of the Poisson bracket $\{$,$\} and the Hamiltonian H$ as

$$
\frac{d f}{d t}=\{H, f\}
$$


We assume that there exists an $n \times n$ matrix valued function $A=\left(a_{i j}\right)_{i, j}$ such that

(i) $\{A \otimes A\}:=\left(\left\{a_{i j}, a_{k l}\right\}\right)_{i k, j l}=[r, A \otimes 1+1 \otimes A]$,

(ii) $H \stackrel{\prime}{=} 1 / N \operatorname{Tr} A^{N}$.

Here $r$ is an $n^{2} \times n^{2}$ constant matrix called the classical $r$ matrix, which satisfies the classical Yang-Baxter equation:

$$
\left[r_{12}, r_{23}\right]+\left[r_{13}, r_{23}\right]+\left[r_{12}, r_{13}\right]=0
$$

where $r_{i j}$ 's are defined as follows: if $r=\sum r_{a} \otimes r^{a}$, then

$$
r_{12}=\sum r_{a} \otimes r^{a} \otimes 1, \quad r_{23}=\sum 1 \otimes r_{a} \otimes r^{a}, \quad r_{13}=\sum r_{a} \otimes 1 \otimes r^{a}
$$

(i) yields

$$
\left\{\operatorname{Tr} A^{m}, A\right\}=\left[m \operatorname{Tr}_{1}\left(\left(A^{m-1} \otimes 1\right) r\right), A\right],
$$

where $\operatorname{Tr}_{1}$ indicates trace on the indices of the first entry and Poisson bracket of the left-hand side is taken elementwise. Directly from (2.1) we obtain for all $k, l$,

$$
\left\{\operatorname{Tr} A^{k}, \operatorname{Tr} A^{l}\right\}=0 .
$$

Consequently time evolution of $A$ is expressed by the Lax representation

$$
\frac{d A}{d t}=[B, A] \quad \text { with } \quad B=\operatorname{Tr}_{1}\left(\left(A^{N-1} \otimes 1\right) r\right),
$$

because of (ii), and $H_{m}=\operatorname{Tr} A^{m+1} /(m+1)$ are shown to be constants of motion commuting with each other. This allows us to consider commuting time flows with respect to Hamiltonian $H_{m}$ and corresponding time variable $t_{m}$, and we get a system of evolution equations

$$
\frac{\partial A}{\partial t_{m}}=\left[B_{m}, A\right] \quad \text { with } \quad B_{m}=\operatorname{Tr}_{1}\left(\left(A^{m} \otimes 1\right) r\right) .
$$

For the Toda molecule (i.e. the finite Toda lattice with free boundary) we can set

$$
A=\left(\begin{array}{ccccc}
\ddots & \ddots & & & 0 \\
\ddots & \frac{1}{2} \partial_{t_{1}} u(-1) & e^{(u(0)-u(-1)) / 2} & & \\
& e^{(u(0)-u(-1)) / 2} & \frac{1}{2} \partial_{t_{1}} u(0) & e^{(u(1)-u(0)) / 2} & \\
& & e^{(u(1)-u(0)) / 2} & \frac{1}{2} \partial_{t_{1}} u(1) & \ddots \\
0 & & & \ddots & \ddots
\end{array}\right)
$$

as stated in Sect. 1 and

$$
H=H_{1}=\frac{1}{2} \operatorname{Tr} A^{2}, \quad r=\sum_{i>j}\left(E_{i j} \otimes E_{j i}-E_{j i} \otimes E_{i j}\right)
$$

For the infinite Toda lattice the trace diverges, as $A$ is an infinite matrix. (As the system has infinite size, total energy, for example, diverges inevitably.) Nevertheless, applying the above argument formally, we can obtain a system of evolution equations of type (2.4). That is to say, $(2.4)(m=1,2, \ldots)$ can be regarded as a system with infinitely many time variables $t_{m}$ and corresponding Hamiltonians $H_{m}$. 
We take (2.4) as the starting point of the following discussion.

2. The 1-Dimensional TL Hierarchy of $r$ Matrix Type. Let $Q$ be the following $\mathbf{Z} \times \mathbf{Z}$ "tri-ogonal" symmetric matrix valued function of $t=\left(t_{1}, t_{2}, \ldots\right)$ :

$$
\begin{gathered}
Q=\operatorname{diag}[a(s)] \Lambda+\operatorname{diag}[b(s)]+\Lambda^{-1} \operatorname{diag}[a(s)], \\
\text { with } a(s)=a(s ; t) \neq 0, \quad b(s)=b(s ; t) .
\end{gathered}
$$

Set

$$
Y_{n}:=\left(Q^{n}\right)_{+}-\left(Q^{n}\right)_{-}, \quad n=1,2, \ldots
$$

The 1-dimensional TL hierarchy of $r$ matrix type is, by definition, the system

$$
\frac{\partial Q}{\partial t_{n}}=\left[Y_{n}, Q\right], \quad n=1,2, \ldots
$$

This corresponds to the system in Sect. 2.1 under the identification

- $A=Q$ with $a(s)=e^{(u(s+1)-u(s)) / 2}, b(s)=\partial_{t_{1}} u(s) / 2$,

- $H_{n}=\operatorname{Tr} Q^{n+1} /(n+1)$,

- $B_{n}=Y_{n}$,

- $r=\sum_{i>j}\left(E_{i j} \otimes E_{j i}-E_{j i} \otimes E_{i j}\right)$.

The equation (2.7) $n=1$ is the original Toda lattice equation.

Now we discuss the Zakharov-Shabat representation, the linearization and the bilinear relations of this system, following the same strategy as that in [U-T].

Proposition 2.1. The 1-dimensional TL hierarchy of $r$ matrix type (2.7) is equivalent to the Zakharov-Shabat representation

$$
\frac{\partial Y_{n}}{\partial t_{m}}-\frac{\partial Y_{m}}{\partial t_{n}}+\left[Y_{n}, Y_{m}\right]=0
$$

Proof. Set

$$
\begin{aligned}
\omega & :=\sum_{n=1}^{\infty} Q^{n} d t_{n}, \\
\Omega_{ \pm} & :=(\omega)_{ \pm}, \\
\Omega_{0} & :=(\omega)_{0}, \\
\xi & :=\sum_{n=1}^{\infty} Y_{n} d t_{n}=\Omega_{+}-\Omega_{-} .
\end{aligned}
$$

Since $Q$ is symmetric, $\Omega_{-}={ }^{t} \Omega_{+}$. Equation (2.7) is rewritten as

$$
d_{t} \omega=[\xi, \omega]_{+}(=\xi \wedge \omega+\omega \wedge \xi),
$$

hence (2.7) is equivalent to

$$
\begin{aligned}
d_{t} \Omega_{0} & =0 \\
d_{t} \Omega_{+} & =2 \Omega_{+} \wedge \Omega_{+}+\Omega_{+} \wedge \Omega_{0}+\Omega_{0} \wedge \Omega_{+}
\end{aligned}
$$


The second equation of (2.9) is equivalent to

$$
\begin{array}{r}
\frac{\partial Q_{+}^{n}}{\partial t_{m}}-\frac{\partial Q_{+}^{m}}{\partial t_{n}}=2\left[Q_{+}^{m}, Q_{+}^{n}\right]+\left[Q_{+}^{m}, Q_{0}^{n}\right]+\left[Q_{0}^{m}, Q_{+}^{n}\right], \\
-\frac{\partial Q_{-}^{n}}{\partial t_{m}}+\frac{\partial Q_{-}^{m}}{\partial t_{n}}=2\left[Q_{-}^{m}, Q_{-}^{n}\right]+\left[Q_{-}^{m}, Q_{0}^{n}\right]+\left[Q_{0}^{m}, Q_{-}^{n}\right] .
\end{array}
$$

Here we abbreviated $\left(Q^{n}\right)_{ \pm}$as $Q_{ \pm}^{n}$. The identity

$$
\begin{aligned}
0=\left[Q^{m}, Q^{n}\right]= & {\left[Q_{+}^{m}, Q_{+}^{n}\right]+\left[Q_{+}^{m}, Q_{0}^{n}\right] } \\
& +\left[Q_{+}^{m}, Q_{-}^{n}\right]+\left[Q_{0}^{m}, Q_{+}^{n}\right]+\left[Q_{0}^{m}, Q_{-}^{n}\right] \\
& +\left[Q_{-}^{m}, Q_{+}^{n}\right]+\left[Q_{-}^{m}, Q_{0}^{n}\right]+\left[Q_{-}^{m}, Q_{-}^{n}\right]
\end{aligned}
$$

yields

$$
\begin{aligned}
{\left[Y_{m}, Y_{n}\right]=} & 2\left[Q_{+}^{m}, Q_{+}^{n}\right]+2\left[Q_{-}^{m}, Q_{-}^{n}\right] \\
& +\left[Q_{+}^{m}, Q_{0}^{n}\right]+\left[Q_{0}^{m}, Q_{+}^{n}\right] \\
& +\left[Q_{0}^{m}, Q_{-}^{n}\right]+\left[Q_{-}^{m}, Q_{0}^{n}\right]
\end{aligned}
$$

Adding the two equations of (2.10), we obtain, using the above identity,

$$
\frac{\partial Y_{n}}{\partial t_{m}}-\frac{\partial Y_{m}}{\partial t_{n}}+\left[Y_{n}, Y_{m}\right]=0 .
$$

Next we assume (2.8). Comparing the upper and lower triangular parts of both sides of (2.8), equivalence of (2.8) and (2.10) can be shown by the same argument as above. Using $Q_{+}^{m}=Q^{m}-Q_{-}^{m}-Q_{0}^{m}$, from (2.10) we see

$$
\begin{gathered}
\partial_{t_{n}} Q^{m}-\left[Y_{n}, Q^{m}\right]=\partial_{t_{m}} Q_{+}^{n}+\partial_{t_{m}} Q_{-}^{n}+\partial_{t_{n}} Q_{0}^{m}+\left[Q^{n}, Q_{+}^{m}\right]+\left[Q_{-}^{m}, Q^{n}\right] \\
=\partial_{t_{m}} Q_{+}^{n}+\partial_{t_{m}} Q_{-}^{n}+\partial_{t_{n}} Q_{0}^{m}+\left[Q^{n},-Q_{-}^{m}-Q_{0}^{m}\right]+\left[Q_{-}^{m}, Q^{n}\right] .
\end{gathered}
$$

Since all the matrices in the right-hand side are of order less than $n$, the order of the left-hand side should be bounded for fixed $n$;

$$
\operatorname{ord}\left(\partial_{t_{n}} Q^{m}-\left[Y_{n}, Q^{m}\right]\right) \leqq n \text {. }
$$

If $\partial_{t_{n}} Q^{m}-\left[Y_{n}, Q^{m}\right] \neq 0$, then it is easy to see that

$$
\operatorname{ord}\left(\partial_{t_{n}} Q^{m}-\left[Y_{n}, Q^{m}\right]\right) \rightarrow+\infty \quad(m \rightarrow+\infty)
$$

which contradicts (2.11). Thus we have proved

$$
\partial_{t_{n}} Q^{m}=\left[Y_{n}, Q^{m}\right]
$$

for all $m$.

In the above proof "order" of a matrix $A=\sum_{j \in \mathbf{Z}} \operatorname{diag}\left[a_{j}(s)\right] \Lambda^{j}$ means

$$
\operatorname{ord} A:=\max \left\{j \in \mathbf{Z} \mid\left(a_{j}(s)\right)_{s \in \mathbf{Z}} \neq 0\right\} \text {. }
$$

\section{Proposition 2.2.}

(i) Suppose $Q$ is a solution of the 1-dimensional TL hierarchy of $r$ matrix type. The 
linear problem for a $\mathbf{Z} \times \mathbf{Z}$ matrix valued function $V(t)$

$$
\begin{aligned}
Q V(t) & =V(t)\left(\Lambda+\Lambda^{-1}\right), \\
\frac{\partial}{\partial t_{n}} V(t) & =Y_{n} V(t), \quad n=1,2, \ldots,
\end{aligned}
$$

has a solution $V(t)=V^{(\infty)}(t)$ of the form

$$
\begin{aligned}
V^{(\infty)}(t) & =\hat{V}(t) \exp \left(\sum_{n=1}^{\infty} t_{n} \varphi_{n}(\Lambda)\right), \\
\hat{V}(t) & =\sum_{j=0}^{\infty} \operatorname{diag}\left[\hat{v}_{j}(s ; t)\right] \Lambda^{-j}, \quad \text { with } \quad \hat{v}_{0}(s) \neq 0,
\end{aligned}
$$

where

$$
\varphi_{n}(\Lambda)=\left(\Lambda+\Lambda^{-1}\right)_{+}^{n}-\left(\Lambda+\Lambda^{-1}\right)_{-}^{n}=\sum_{k=0}^{n} \operatorname{sgn}(2 k-n)\left(\begin{array}{l}
n \\
k
\end{array}\right) \Lambda^{2 k-n}
$$

(ii) Then $V^{(0)}(t)={ }^{t}\left(V^{(\infty)}(t)\right)^{-1}={ }^{t} \hat{V}(t)^{-1} \exp \left(\sum_{n=1}^{\infty} t_{n} \varphi_{n}(\Lambda)\right)$ is also a solution of $(2.12)$.

Proof.

(i) First we rewrite (2.12) into the linear problem of $\hat{V}(t)$;

$$
\begin{aligned}
Q \hat{V}(t) & =\hat{V}(t)\left(\Lambda+\Lambda^{-1}\right) \\
\frac{\partial}{\partial t_{n}} \hat{V}(t) & =Y_{n} \hat{V}(t)-\hat{V}(t) \varphi_{n}(\Lambda), \quad n=1,2, \ldots
\end{aligned}
$$

It is also written as

$$
\begin{aligned}
Q \hat{V}(t) & =\hat{V}(t)\left(\Lambda+\Lambda^{-1}\right) \\
\frac{\partial}{\partial t_{n}} \hat{V}(t) & =\left(Y_{n}-Q^{n}\right) \hat{V}(t)+\hat{V}(t)\left(\left(\Lambda+\Lambda^{-1}\right)^{n}-\varphi_{n}(\Lambda)\right)
\end{aligned}
$$

By the assumption $a(s) \neq 0$ in (2.5), we can prove by induction that there exists a invertible lower triangular matrix $\hat{V}_{0}$ satisfying

$$
Q \hat{V}_{0}=\hat{V}_{0}\left(\Lambda+\Lambda^{-1}\right) \text {. }
$$

Let us consider the Cauchy problem

$$
\begin{aligned}
& \frac{\partial}{\partial t_{n}} \hat{V}=-\left(Q_{0}^{n}+2 Q_{-}^{n}\right) \hat{V}+\hat{V}\left(\left(\Lambda+\Lambda^{-1}\right)_{0}^{n}+2\left(\Lambda+\Lambda^{-1}\right)_{-}^{n}\right), \\
& \left.\hat{V}\right|_{t=0}=\left.\hat{V}_{0}\right|_{t=0} .
\end{aligned}
$$

The first equation of (2.14) is nothing but the second equation of $\left(2.12^{\prime \prime}\right)$. Thanks to (2.7) and (2.8),

$$
\left[\frac{\partial}{\partial t_{n}}+\left(Q^{n}-Y_{n}\right), \frac{\partial}{\partial t_{m}}+\left(Q^{m}-Y_{m}\right)\right]=0
$$


hence $(2.14)$ is a compatible system. Since all coefficients of $\hat{V}(t)$ in (2.14) are lower triangular, (2.14) has a unique solution of a lower triangular matrix. That solution satisfies

$$
\begin{aligned}
\frac{\partial}{\partial t_{n}}\left(Q \hat{V}-\hat{V}\left(\Lambda+\Lambda^{-1}\right)\right)= & \left(Y_{n}-Q^{n}\right)\left(Q \hat{V}-\hat{V}\left(\Lambda+\Lambda^{-1}\right)\right) \\
& +\left(Q \hat{V}-\hat{V}\left(\Lambda+\Lambda^{-1}\right)\right)\left(\left(\Lambda+\Lambda^{-1}\right)^{n}-\varphi_{n}(\Lambda)\right)
\end{aligned}
$$

i.e. $\left(Q \hat{V}-\hat{V}\left(\Lambda+\Lambda^{-1}\right)\right)$ is a solution of the Cauchy problem (2.14) with the initial value

$$
\left.\left(Q \hat{V}-\hat{V}\left(\Lambda+\Lambda^{-1}\right)\right)\right|_{t=0}=\left.\left(Q \hat{V}_{0}-\hat{V}_{0}\left(\Lambda+\Lambda^{-1}\right)\right)\right|_{t=0}=0 .
$$

From the uniqueness of the solution we see

$$
Q \hat{V}(t)=\hat{V}(t)\left(\Lambda+\Lambda^{-1}\right)
$$

for all $t$. It is clear from (2.14) that

$$
\frac{\partial}{\partial t_{n}} \hat{v}_{0}(s ; t)=\text { some function } \times \hat{v}_{0}(s ; t) .
$$

Hence

$$
\hat{v}_{0}(s ; t)=\hat{v}_{0}(s ; 0) \times \exp (\text { some function) }
$$

Since $\hat{v}_{0}(s ; 0) \neq 0$ by the initial condition of $\hat{V}(t)$,

$$
\hat{v}_{0}(s ; t) \neq 0
$$

for all $t$. So we have obtained the desired solution of $\left(2.12^{\prime \prime}\right)$, i.e. (2.12).

(ii) By the symmetry of $Q$ and $\Lambda+\Lambda^{-1}$ and the anti-symmetry of $Y_{n}$ and $\varphi_{n}(\Lambda)$, transposing $\left(2.12^{\prime}\right)$, we get

$$
\begin{aligned}
Q^{t} \hat{V}^{-1}(t) & ={ }^{t} \hat{V}^{-1}(t)\left(\Lambda+\Lambda^{-1}\right), \\
\frac{\partial}{\partial t_{n}}{ }^{t} \hat{V}^{-1}(t) & =Y_{n}{ }^{t} \hat{V}^{-1}(t)-{ }^{t} \hat{V}^{-1}(t) \varphi_{n}(\Lambda), \quad n=1,2, \ldots
\end{aligned}
$$

This shows that $V^{(0)}$ is also a solution of (2.12).

As in the case of 1-dimensional TL hierarchy of Ueno and Takasaki, that of $r$ matrix type can be characterized by the bilinear relations of these wave matrices $V^{\left(\begin{array}{c}0 \\ \infty\end{array}\right)}$

\section{Theorem 2.3.}

(i) $V^{\left(\begin{array}{l}0 \\ \infty\end{array}\right)}(t)$ in Proposition 2.2 satisfy the following condition;

$$
\partial_{t}^{\alpha} V^{(\infty)}(t) V^{(\infty)}(t)^{-1}=\partial_{t}^{\alpha} V^{(0)}(t) V^{(0)}(t)^{-1},
$$

for all multi-indices $\alpha=\left(\alpha_{1}, \alpha_{2}, \ldots\right)$. Or, equivalently,

$$
V^{(\infty)}(t) V^{(\infty)}\left(t^{\prime}\right)^{-1}=V^{(0)}(t) V^{(0)}\left(t^{\prime}\right)^{-1}
$$

for all $t$ and $t^{\prime}$. 
(ii) Conversely, if $V^{(\infty)}(t)$ of the form (2.13) and

$$
V^{(0)}:={ }^{t} V^{(\infty)}(t)^{-1}
$$

satisfy (2.15) or (2.16), and if

$$
V^{(\infty)}(t)\left(\Lambda+\Lambda^{-1}\right) V^{(\infty)}(t)^{-1}=V^{(0)}(t)\left(\Lambda+\Lambda^{-1}\right) V^{(0)}(t)^{-1}(=: Q)
$$

holds, then $Q$ is a solution of the 1-dimensional TLH of $r$ matrix type and $V^{\left(\begin{array}{c}0 \\ \infty\end{array}\right)}(t)$ are wave matrices corresponding to it.

Proof.

(i) This statement can be easily deduced from

$$
\begin{aligned}
\partial_{t_{n}} V^{(\infty)}(t) V^{(\infty)}(t)^{-1} & =\partial_{t_{n}} V^{(0)}(t) V^{(0)}(t)^{-1} \\
& =Y_{n}, \quad n=1,2, \ldots
\end{aligned}
$$

by induction (cf. [U-T ] (1.2.17), (1.2.18)).

(ii) The assumption (2.15) and (2.16) are equivalent to (2.18) as shown in the proof of (i). We set $Y_{n}$ as in (2.18). From (2.13) we obtain for all $n$,

$$
\begin{aligned}
\partial_{t_{n}} V^{(\infty)} V^{(\infty)-1} & =\partial_{t_{n}} \hat{V} \hat{V}^{-1}+\hat{V} \varphi_{n}(\Lambda) \hat{V}^{-1}, \\
\partial_{t_{n}} V^{(0)} V^{(0)-1} & =-{ }^{t} \hat{V}^{-1} \partial_{t_{n}} \hat{V}+{ }^{t} \hat{V}^{-1} \varphi_{n}(\Lambda)^{t} \hat{V}
\end{aligned}
$$

Hence

$$
\begin{aligned}
Y_{n} & =\partial_{t_{n}} \hat{V} \hat{V}^{-1}+\hat{V} \varphi_{n}(\Lambda) \hat{V}^{-1} \\
& =-{ }^{t} \hat{V}^{-1} \partial_{t_{n}} \hat{V}+{ }^{t} \hat{V}^{-1} \varphi_{n}(\Lambda)^{t} \hat{V}
\end{aligned}
$$

which shows

$$
{ }^{t} Y_{n}=-Y_{n} .
$$

Noting that $\hat{V}$ is lower triangular, we get

$$
\begin{aligned}
\left(Y_{n}\right)_{+} & =\left(\partial_{t_{n}} \hat{V} \hat{V}^{-1}\right)_{+}+\left(\hat{V} \varphi_{n}(\Lambda) \hat{V}^{-1}\right)_{+} \\
& =\left(\hat{V} \varphi_{n}(\Lambda) \hat{V}^{-1}\right)_{+} \\
& =\left(\hat{V} \varphi_{n}(\Lambda)_{+} \hat{V}^{-1}\right)_{+}=\left(\hat{V}\left(\Lambda+\Lambda^{-1}\right)_{+}^{n} \hat{V}^{-1}\right)_{+} \\
& =\left(\hat{V}\left(\Lambda+\Lambda^{-1}\right)^{n} \hat{V}^{-1}\right)_{+} .
\end{aligned}
$$

This formula and

$$
Q=\hat{V}\left(\Lambda+\Lambda^{-1}\right) \hat{V}^{-1}
$$

imply

$$
\left(Y_{n}\right)_{+}=\left(Q^{n}\right)_{+} \cdot
$$

Since assumption (2.17) means that $Q$ is symmetric, from (2.20) and (2.22) we obtain $Y_{n}=\left(Q^{n}\right)_{+}-\left(Q^{n}\right)_{-}$. It is easily seen from (2.19) that

$$
\partial_{t_{n}} V^{\left(\begin{array}{c}
0 \\
x
\end{array}\right)}(t)=Y_{n} V^{\left(\begin{array}{c}
0 \\
x
\end{array}\right)}(t)
$$


Finally, (2.21) and the symmetry of $Q$ assures that $Q$ is expressed as

$$
Q=\operatorname{diag}[a(s)] \Lambda+\operatorname{diag}[b(s)]+\Lambda^{-1} \operatorname{diag}[a(s)],
$$

where $a(s) \neq 0$ for all $s$.

3. Comparison with the Result of Sect. 1. In Sect. 1.2 we have seen that the solution of the 1-dimensional TL hierarchy of [U-T] is determined by wave matrices which satisfy the bilinear relations; i.e.

(I) An invertible lower triangular matrix $\hat{V}(t)$.

(II) $V^{(\infty)}(t)=\hat{V}(t) \exp \left(\sum_{n=1}^{\infty} t_{n}\left(\Lambda^{n}-\Lambda^{-n}\right)\right), V^{(0)}(t)={ }^{t} V^{(\infty)}(t)^{-1}$.

(III) $V^{(\infty)}(t) V^{(\infty)}\left(t^{\prime}\right)^{-1}=V^{(0)}(t) V^{(0)}\left(t^{\prime}\right)^{-1}$.

(IV) $V^{(\infty)}(t)\left(\Lambda+\Lambda^{-1}\right) V^{(\infty)}(t)^{-1}=V^{(0)}(t)\left(\Lambda+\Lambda^{-1}\right) V^{(0)}(t)^{-1}$.

On the other hand, in Sect. 2.2 it was shown that the solution of the 1-dimensional TL hierarchy of $r$ matrix type is also determined by the following data (in order to avoid confusion with the above hierarchy, time variables are denoted by $\left.s=\left(s_{1}, s_{2}, \ldots\right)\right)$;

$\left(\mathrm{I}^{\prime}\right)$ An invertible lower triangular matrix $\hat{V}(s)$.

$\left(\mathrm{II}^{\prime}\right) V^{(\infty)}(s)=\hat{V}(s) \exp \left(\sum_{n=1}^{\infty} s_{n} \varphi_{n}(\Lambda)\right), V^{(0)}(s)={ }^{t} V^{(\infty)}(s)^{-1}$

$\left(\mathrm{III}^{\prime}\right) V^{(\infty)}(s) V^{(\infty)}\left(s^{\prime}\right)^{-1}=V^{(0)}(s) V^{(0)}\left(s^{\prime}\right)^{-1}$.

$\left(\mathrm{IV}^{\prime}\right) V^{(\infty)}(s)\left(\Lambda+\Lambda^{-1}\right) V^{(\infty)}(s)^{-1}=V^{(0)}(s)\left(\Lambda+\Lambda^{-1}\right) V^{(0)}(s)^{-1}$.

Now it is obvious that the two 1-dimensional TL hierarchy are identical to each other under the identification of time variables

$$
\sum_{n=1}^{\infty} t_{n}\left(\lambda^{n}-\lambda^{-n}\right)=\sum_{n=1}^{\infty} s_{n} \varphi_{n}(\lambda)
$$

where $\varphi_{n}(\lambda)=\sum_{k=1}^{n} \operatorname{sgn}(2 k-n)\left(\begin{array}{l}n \\ k\end{array}\right) \lambda^{2 k-n}$. In other words, $t$ and $s$ are written as a linear combination of each other and setting $\mathbf{t}={ }^{t}\left(t_{1}, t_{2}, \ldots\right)$ and $\mathbf{s}={ }^{t}\left(s_{1}, s_{2}, \ldots\right)$, the relation can be written explicitly as

$$
\mathbf{t}=\mathbf{C s}
$$

where

$$
\begin{aligned}
\mathbf{C} & =\left(C_{n m}\right)_{n, m \in \mathbf{N}}, \\
C_{n m} & = \begin{cases}\left(\begin{array}{c}
m \\
(m+n) / 2
\end{array}\right), & \text { if } n \leqq m \text { and } n \equiv m(\bmod 2), \\
0, & \text { otherwise. }\end{cases}
\end{aligned}
$$

Hence if we set

then

$$
\vec{\partial}_{t}=\left(\partial_{t_{1}}, \partial_{t_{2}}, \ldots\right), \quad \vec{\partial}_{s}=\left(\partial_{s_{1}}, \partial_{s_{2}}, \ldots\right)
$$

$$
\vec{\partial}_{s}=\vec{\partial}_{t} \mathbf{C}
$$


As for the $r$ matrix type there is a correspondence $\partial_{s_{n}}=\left\{H_{n}, \cdot\right\}$. By the above equation we obtain (formal) Hamiltonian $\widetilde{H}_{n}$ corresponding to time $t_{n}$ as follows;

$$
\begin{gathered}
\left(\tilde{H}_{1}, \tilde{H}_{2}, \ldots\right)=\left(H_{1}, H_{2}, \ldots\right) C^{-1}, \\
H_{n}=\frac{1}{n+1} \operatorname{Tr} Q(s)^{n+1}=\frac{1}{n+1} \operatorname{Tr} Q_{1}(t)^{n+1} .
\end{gathered}
$$

So far the result concerns the 1-dimensional TL hierarchy.

\section{Hamiltonian Structure of TL Hierarchy}

1. Review of the Toda Lattice Field Theories of Olive and Turok (I). Here and in the subsequent section we recall the theory originated from the quantum inverse scattering method and applied to the Toda lattice by Olive and Turok [O-T2,3]. In this section the generalized 2-dimensional Toda lattice of Mikhailov, Olshanetsky and Perelomov [M-O-P] associated to an affine algebra $g$ is transformed into an equation in the principal subalgebra $\mathfrak{s}$ of $\mathfrak{g}$ by a special gauge transformation. The transformed gauge potential makes it easy to write down the transition matrix of the space direction and gives the conserved current, because of the abelian property of $\mathfrak{s}$. We generalize the theory to all affine Kac-Moody algebras, while Olive and Turok worked only on the non-twisted case.

For terms of the theory of Kac-Moody algebras we refer to the standard textbook [Ka], but to fix the notations we briefly recall what is needed. Let $g=X_{N}^{(\kappa)}$ be an affine Lie algebra. We mainly use the following principal realization:

$$
\mathfrak{g}=\bigoplus_{j \in \mathbf{Z}} \lambda^{j} \stackrel{\mathfrak{g}}{j}_{j} \oplus \mathbf{C} c \oplus \mathbf{C d},
$$

where $\stackrel{\mathfrak{g}}{=}=X_{N}$, a finite dimensional simple Lie algebra and $\stackrel{\circ}{\mathfrak{g}}=\bigoplus \stackrel{\mathfrak{g}}{j}^{-}$is its $h^{(\kappa)}\left(\kappa\right.$-Coxeter number) gradation. The Cartan subalgebra of $\mathfrak{g}$ is $\mathfrak{h}=\stackrel{\circ}{g}_{0} \oplus \mathbf{C} c \oplus \mathbf{C d}$.

The Lie algebra, on which the whole theory lives, is

$$
\overline{\mathfrak{g}}:=[\mathfrak{g}, \mathfrak{g}] / \mathbf{C} c \cong \bigoplus_{j \in \mathbf{Z}} \overline{\mathfrak{g}}_{j}, \quad \overline{\mathfrak{g}}_{j}=\lambda^{j} \stackrel{\mathfrak{g}}{\bar{j}}_{\bar{j}}
$$

and decomposed as follows:

$$
\begin{aligned}
& \overline{\mathfrak{g}}=\overline{\mathfrak{h}} \oplus \mathfrak{n}_{+} \oplus \mathfrak{n}_{-}, \\
& \overline{\mathfrak{h}}:=\stackrel{\mathfrak{g}}{0}_{0}=\text { Cartan subalgebra of } \stackrel{\mathfrak{g}}{ }, \\
& \mathfrak{n}_{ \pm}:=\bigoplus_{j \lessgtr 0} \overline{\mathfrak{g}}_{j} .
\end{aligned}
$$

$\Lambda_{+}=\sum_{i=0}^{l} \bar{e}_{i}$ and $\Lambda_{-}=\sum_{i=0}^{l} \bar{f}_{i}$ are called cyclic elements of $\overline{\mathfrak{g}}$, where $e_{i}$ and $f_{i}(i=0, \ldots, l=$ rank of $\mathfrak{g})$ are Chevalley generators of $\mathfrak{g}$ and $\bar{x}$ is the image of $x \in \mathfrak{g}$ in $\overline{\mathrm{g}}$. The subalgebra of $\overline{\mathrm{g}}$,

$$
\overline{\mathfrak{s}}=\left\{x \in \overline{\mathfrak{g}} \mid\left[x, \Lambda_{+}\right]=0\right\}=\left\{x \in \overline{\mathfrak{g}} \mid\left[x, \Lambda_{-}\right]=0\right\},
$$

is abelian and called the principal subalgebra of $\overline{\mathrm{g}}$ (cf. [Ka] Chap. 14). $\overline{\mathfrak{s}}$ is graded:

$$
\overline{\mathfrak{s}}=\bigoplus_{j \in \mathbf{Z}} \overline{\mathfrak{s}}_{j} \quad \overline{\mathfrak{s}}_{j}=\overline{\mathfrak{s}} \cap \overline{\mathfrak{g}}_{j}
$$


and the integers of the set which contains $j$ with multiplicity $\operatorname{dim} \overline{\mathfrak{s}}_{j}$ are called exponents. $\operatorname{dim} \overline{\mathfrak{s}}_{j} \leqq 1$ in all cases but one: $\operatorname{dim} \overline{\mathfrak{s}}_{j}=2$ when $\mathfrak{g}=D_{2 m}^{(1)}$ and $j \equiv 2 m-1$ $(\bmod 2 m-2)$.

Hereafter we restrict ourselves to the cases $\operatorname{dim} \overline{\mathfrak{s}}_{j} \leqq 1$ for all $j$ and denote the set of positive (respectively negative) exponents as $E_{+}$(respectively $E_{-}$). Fix the basis of $\overline{\mathfrak{s}}_{j}=\mathbf{C} \Lambda_{j}\left(j \in E_{ \pm}\right)$so that $\left(\Lambda_{j} \mid \Lambda_{k}\right)=\delta_{j+k 0} .(\cdot \mid \cdot)$ denotes the canonical invariant bilinear form of $\mathfrak{g}$, which is non-degenerate on $\overline{\mathfrak{g}}$. (Excluding the case $\mathfrak{g}=D_{2 m}^{(1)}$ is not essential at all, but only for the sake of simplicity of notations.)

The generalized Toda lattice equation in the sense of $[\mathrm{M}-\mathrm{O}-\mathrm{P}]$ is

$$
\left[\partial_{z}-Q, \partial_{t}-X\right]=0
$$

of the unknown function $u(z, t) \in \overline{\mathfrak{h}}$, where

$$
\begin{aligned}
& Q=\frac{\partial_{t} u}{2}+e^{\mathrm{ad} u / 2} \Lambda_{+}+e^{-\mathrm{ad} u / 2} \Lambda_{-}, \\
& X=\frac{\partial_{z} u}{2}+e^{\mathrm{ad} u / 2} \Lambda_{+}-e^{-\mathrm{ad} u / 2} \Lambda_{-} .
\end{aligned}
$$

The class of functions considered is a suitable subring $\mathscr{A}=\mathscr{A}_{z}$ of functions (or formal power series) of $\zeta=(\pi / L) z$ and $t$, closed under differentiation and exponentiation. Here $L$ is a (formal) parameter designating the period of the system, i.e. we impose on $u$ the periodic condition in $z$ :

$$
u(z, t)=u(z+2 L, t) \quad u(\zeta, t)=u(\zeta+2 \pi, t) .
$$

$u$ is also regarded as a function on a cylinder $\mathbf{S}^{1} \times \mathbf{R}$.

The solution $\mathbf{T}\left(z^{1}, z^{2}, t\right)$ of the following Cauchy problem is called the transition matrix from $z^{1}$ to $z^{2}$ :

$$
\begin{aligned}
\frac{\partial}{\partial z^{1}} \mathbf{T}\left(z^{1}, z^{2}\right) & =Q\left(z^{1}\right) \mathbf{T}\left(z^{1}, z^{2}\right), \\
\frac{\partial}{\partial z^{2}} \mathbf{T}\left(z^{1}, z^{2}\right) & =-\mathbf{T}\left(z^{1}, z^{2}\right) Q\left(z^{2}\right), \\
\mathbf{T}(z, z) & =1 .
\end{aligned}
$$

The monodromy matrix $\mathbf{T}_{L}$ is defined as

$$
\mathbf{T}_{L}=\mathbf{T}\left(z^{1}=-L, z^{2}=L\right)=\mathbf{T}\left(\zeta^{1}=-\pi, \zeta^{2}=\pi\right)
$$

which plays a central role in the quantum inverse scattering method.

The main goal of this section is to give the explicit form for the transition and monodromy matrices, making use of "abelianization," a special kind of gauge transformation.

Remark 3.1. $\mathbf{T}\left(z^{1}, z^{2}, t\right)$ is considered as an element of $\mathscr{A}_{z^{1}} \otimes \mathscr{A}_{z^{2}} \otimes \overline{U(\overline{\mathfrak{g}})}$, where $\overline{U(\overline{\mathfrak{g}})}$ is formal completion of the universal enveloping algebra of $\overline{\mathrm{g}}$. But we can use any $\mathbf{Z}$ graded associative algebra containing $\overline{\mathfrak{g}}$ and its appropriate completion instead of $\overline{U(\overline{\mathfrak{g}})}$. 
Now we define several gauge groups acting on $\overline{(\overline{\mathfrak{g}})}=($ formal completion of $\overline{\mathfrak{g}})$ :

$$
\begin{aligned}
H & :=\left\{\exp H_{1} \cdots \exp H_{n} \mid n \in \mathbf{N} H_{i} \in \overline{\mathfrak{h}}\right\} \subset \mathbf{C}[[\overline{\mathfrak{h}}]], \\
N_{ \pm} & :=\left\{\exp X_{1} \cdots \exp X_{n} \mid n \in \mathbf{N} X_{i} \in \mathfrak{n}_{ \pm}\right\} \subset \overline{U\left(\mathfrak{n}_{ \pm}\right)} .
\end{aligned}
$$

Let $G=H$ or $N_{ \pm}$and $A(z, t)$ be a function with values in $\overline{(\overline{\mathrm{g}})}$. When $A(z, t)$ is regarded as a gauge potential with respect to $z$ (e.g. $Q$ in the Toda lattice), gauge transformation of $A$ by $g(z, t) \in G$ is defined as follows:

$$
A^{g}(z, t):=\operatorname{Ad}\left(g^{-1}\right) A(z, t)+\partial_{z} g^{-1}(z, t) g(z, t) \in \overline{(\overline{\mathfrak{g}})} .
$$

$A^{g}$ is algebraically well defined if:

- $A$ :arbitrary $\quad ; g \in H$,

- $A \in \bigoplus_{j \leqq N_{0}} \lambda^{j} \stackrel{\mathrm{g}}{j}_{j}$ for some $N_{0} ; g \in N_{-}$,

- $A \in \underset{N_{0} \leqq j}{\bigoplus} \lambda^{j} \mathfrak{g}_{j}$ for some $N_{0} ; \quad g \in N_{+}$.

Example. The gauge potential $Q$ is transformed by $h_{ \pm}=h^{ \pm 1}:=\exp (\mp u(z, t) / 2)$ into

$$
\begin{aligned}
& Q^{h_{+}}=\Lambda_{+}+\frac{1}{2}\left(\partial_{z}+\partial_{t}\right) u+e^{-\mathrm{ad} u} \Lambda_{-}, \\
& Q^{h_{-}}=\Lambda_{-}+\frac{1}{2}\left(\partial_{z}-\partial_{t}\right) u+e^{\mathrm{ad} u} \Lambda_{+} .
\end{aligned}
$$

Definition 3.2. $f \in \mathscr{A}$ is called local if $f$ is a differential polynomial of coordinate component of $u(z, t)$.

Theorem 3.3. (Abelianization [O-T2]) There exist local functions $g_{+}(z, t) \in N_{-}$, $g_{-}(z, t) \in N_{+}$such that

$$
a_{ \pm}(z, t):=\left(Q^{h_{ \pm}}\right)^{g_{ \pm}} \in \overline{\mathfrak{s}} \text {. }
$$

Proof. As $g_{-} \in N_{+}$is determined in the same way, we only show how $g_{+} \in N_{-}$is determined. For simplicity of notations, we drop the sign $+: h=h_{+}, g=g_{+}, a=a_{+}$.

First let $Q_{k}(z, t), a_{k}(z, t)$ be elements of $\overline{\mathfrak{g}}_{k}$ such that

$$
Q^{h}=\sum_{k \leqq 1} Q_{k}, \quad a=\sum_{k \leqq 1} a_{k} .
$$

By (3.2) $Q_{1}=a_{1}=\Lambda_{+} ; Q^{h}=\Lambda_{+}+Q_{0}+Q_{-1}+\cdots, a=\Lambda_{+}+a_{0}+a_{-1}+\cdots$. Take $g(z, t) \in N_{-}$of the form:

$$
g(z, t)=\exp \left(-\sum_{k \leqq-1} X_{k}(z, t)\right), \quad X_{k}(z, t) \in \overline{\mathfrak{g}}_{k} .
$$

Now we determine $X_{k}$ recursively, so that (3.3) holds. By definition

$$
\begin{aligned}
\sum_{k \leqq 1} a_{k}=\left(Q^{h}\right)^{g} & =\exp \left(\operatorname{ad}\left(-\sum_{k \leqq-1} X_{k}\right)\right) Q^{h}+\partial_{z} g^{-1} g \\
& =\exp \left(\operatorname{ad}\left(-\sum_{k \leqq-1} X_{k}\right)\right) Q^{h}+\sum_{k \leqq-1} Y_{k} .
\end{aligned}
$$

Here $Y_{k}$ is a differential polynomial of $X_{-1}, \ldots, X_{k}$. Taking the $\bar{g}_{k}$ component of 
where $\mathbf{T}_{ \pm}$is the solution of the following Cauchy problem:

$$
\begin{aligned}
\frac{\partial}{\partial z^{1}} \mathbf{T}_{ \pm}\left(z^{1}, z^{2}\right) & =a_{ \pm}\left(z^{1}\right) \mathbf{T}_{ \pm}\left(z^{1}, z^{2}\right) \\
\frac{\partial}{\partial z^{2}} \mathbf{T}_{ \pm}\left(z^{1}, z^{2}\right) & =-\mathbf{T}_{ \pm}\left(z^{1}, z^{2}\right) a_{ \pm}\left(z^{2}\right) \\
\mathbf{T}_{ \pm}(z, z) & =1 .
\end{aligned}
$$

Thanks to the abelian property of $a_{ \pm}$, i.e. $a_{ \pm} \in \overline{\mathfrak{s}},(3.6)$ is explicitly solved:

$$
\mathbf{T}_{ \pm}\left(z^{1}, z^{2}\right)=\exp \left(-\int_{z^{1}}^{z^{2}} a_{ \pm}(z) d z\right) \in \overline{U(\overline{\mathfrak{s}})}
$$

Therefore we obtain the transition matrix explicitly from (3.5) and (3.7).

Remark 3.5. $\mathbf{T}_{ \pm}$is algebraically well defined by "rescaling" of $z$,

$$
\mathbf{T}_{ \pm}\left(z^{1}, z^{2}\right)=\exp \left(-\not \int_{\zeta^{1}}^{\zeta^{2}} a_{ \pm}(\zeta) d \zeta\right), \quad \psi=L / \pi,
$$

as a power series of $\ell$.

The importance of $a_{ \pm}$does not consist only in solving (3.1). In the next section we regard $a_{ \pm}$as the generating function of mutually commuting Hamiltonians. Here, before ending this section, we show that this gives conserved currents.

Next lemma is a consequence of Lemma 3.4 and used also in the following section.

Lemma 3.6. ([O-T2]). Let $C$ be such a gauge potential associated to an independent variable $y$ (e.g. $t$ ) that

$$
C(y) \in \bigoplus_{k \leqq N} \mathfrak{g}_{k}, \quad\left(\text { respectively } C(y) \in \underset{k \leqq N}{\bigoplus_{-k}} \mathfrak{g}_{-k}\right),
$$

for $N \gg 0$ and

$$
\begin{array}{r}
{\left[\partial_{z}-a_{+}, \partial_{y}-C\right]=0,} \\
\text { (respectively } \left.\left[\partial_{z}-a_{-}, \partial_{y}-C\right]=0\right) .
\end{array}
$$

Then $C \in \overline{\mathfrak{s}}$.

Proof. Lemma 3.4 yields the decomposition

where

$$
C=C_{\mathrm{s}}+C_{\mathrm{n}},
$$

$$
\begin{gathered}
C_{\mathrm{s}} \in \overline{\mathfrak{s}}, \\
C_{\mathrm{n}}=\sum_{k \leqq N} \lambda^{k} C_{\mathrm{n}, k}, \quad C_{\mathrm{n}, k} \in \mathrm{n}_{\mathrm{S}, k} .
\end{gathered}
$$

Then, from (3.9),

$$
0=\left[\partial_{z}-a_{+}, \partial_{y}-C_{s}\right]-\left[\partial_{z}-a_{+}, C_{n}\right] .
$$


both hand sides, we see that

$$
B_{k}:=a_{k}-\left[X_{k-1}, \Lambda_{+}\right] \quad(k \leqq 0)
$$

is expressed as a differential polynomial of $Q_{0}, \ldots, Q_{k}, X_{-1}, \ldots, X_{k}$.

We need several facts about the principal subalgebra of $\overline{\mathfrak{g}}$. (This was not mentioned by Olive and Turok, but (iii) seems essential.)

\section{Lemma 3.4.}

(i) In the principal realization of $\mathrm{g}=X_{N}^{(\kappa)}$,

$$
\overline{\mathfrak{s}}_{j}=\lambda^{j} S_{\bar{j}}^{(\kappa)}, \quad \Lambda_{j}=\lambda^{j} \stackrel{\Lambda}{j}_{j}
$$

where $S^{(\kappa)}=\bigoplus_{\bar{j} \in \mathbf{Z} / h^{(\kappa)} \mathbf{Z}} S_{j}^{(\kappa)}$ is $\kappa$-principal subalgebra of $\stackrel{\circ}{\mathfrak{g}}, S_{\bar{j}}^{(\kappa)}=S^{(\kappa)} \cap \stackrel{\mathfrak{g}}{j}_{j}$ and $\AA_{j} \in S_{\bar{j}}^{(\kappa)}$. (ii) $S^{(\kappa)}$ is a $\stackrel{\bar{j} \in \mathbf{Z} / h^{(\kappa)} \mathbf{Z}}{C}$ artan subalgebra of $\stackrel{\mathrm{g}}{\mathrm{g}}$. Let $\Delta_{S}$ is the set of roots of $\stackrel{\mathrm{g}}{\mathrm{g}}$ with respect to $S^{(\kappa)}$ and $e_{\alpha}\left(\alpha \in \Delta_{S}\right)$ is root vectors. Then $\stackrel{\mathrm{g}}{\text { is decomposed as }}$

$$
\stackrel{\circ}{\mathrm{g}}=S^{(\kappa)} \oplus \mathrm{n}_{S}, \quad \mathrm{n}_{S}=\bigoplus_{\alpha \in \Delta_{S}} \mathbf{C} e_{\alpha}
$$

(this is obvious), and ad $\AA_{1}$ acts non-degenerately on $n_{S}$.

(iii)

$$
\mathrm{n}_{s}=\bigoplus \mathrm{n}_{s, \bar{j}}, \quad \mathrm{n}_{s, \bar{j}}:=\mathrm{n}_{s} \cap \stackrel{\circ}{\mathfrak{g}}_{j}, \quad \text { and } \quad \stackrel{\circ}{\mathfrak{j}}_{\bar{j}}=S_{\bar{j}}^{(\kappa)} \oplus \mathrm{n}_{s, \bar{j}}
$$

Admitting this lemma, we can set

$$
B_{k}=\lambda^{k}\left(B_{k}\right)_{S}+\lambda^{k}\left(B_{k}\right)_{S^{\perp}} \quad\left(B_{k}\right)_{S} \in S_{\bar{k}}^{(\kappa)}, \quad\left(B_{k}\right)_{S^{\perp} \in \mathfrak{n}_{S, \bar{k}}} .
$$

Being non-degenerate on $n_{S}$, ad $\AA_{1}$ induces a linear isomorphism $n_{S, \bar{k}} \rightarrow n_{s, \overline{k+1}}$,

$$
\begin{aligned}
a_{k} & =\lambda^{k}\left(B_{k}\right)_{S}, \\
X_{k-1} & =\lambda^{k-1}\left(\left.\operatorname{ad} \AA_{1}\right|_{\mathrm{r}_{S . \bar{h}}}\right)^{-1}\left(B_{k}\right)_{S^{\perp}}
\end{aligned}
$$

are the desired solutions of (3.4).

Proof of Lemma 3.4.

(i),(ii) See [Ka] Sect. 14.2.

(iii) There exists an $h^{(\kappa)}$-th order automorphism $\sigma$ such that $\stackrel{\circ}{\mathfrak{g}}=\bigoplus \stackrel{\mathfrak{g}}{j}_{j}$ is its eigenspace decomposition (cf. [Ka] Ch.8). Since $\AA_{1}$ belongs to $\stackrel{\circ}{g}_{1}$, the centralizer of $\Lambda_{1}, S^{(\kappa)}$, is invariant under the action of $\sigma$.

Hence $\sigma\left(e_{\alpha}\right)\left(\alpha \in \Delta_{S}\right)$ is also a root vector with respect to $S^{(\kappa)}$. This means $\mathrm{n}_{S}$ is invariant under the action of $\sigma$. The eigenspace decomposition of $\left.\sigma\right|_{\text {ns }_{s}}$ is

$$
\mathrm{n}_{s}=\oplus \mathrm{n}_{s, \bar{j}}
$$

Using the above gauge transformation, the solution of (3.1), the transition matrix, is written as:

$$
\mathbf{T}\left(z^{1}, z^{2}, t\right)=\tilde{g}_{ \pm}\left(z^{1}, t\right) \mathbf{T}_{ \pm}\left(z^{1}, z^{2}, t\right) \tilde{g}_{ \pm}\left(z^{2}, t\right)^{-1},
$$

with

$$
\tilde{g}(z, t)=h_{ \pm}(z, t) g_{ \pm}(z, t)
$$


The first term belongs to $\overline{\mathfrak{s}}$, while the second belongs to $\bigoplus \lambda^{k} \mathfrak{n}_{s, k}$. Hence

$$
\left[\partial_{z}-a_{+}, C_{n}\right]=0 .
$$

Suppose $C_{\mathrm{n}} \neq 0$ and fix $N$ so that $C_{\mathrm{n}, N} \neq 0$. Since $a_{+}=\Lambda_{+}+\cdots$, the highest degree term of $(3.10)$ is

$$
\left[\Lambda_{+}, C_{n, N}\right]=0 .
$$

By definition of $\overline{\mathfrak{s}}$, this means $C_{n, N} \in \overline{\mathfrak{s}}$, which is contradiction, i.e. $C_{\mathrm{n}}=0, C \in \overline{\mathfrak{s}}$.

For the solution of the Toda lattice equation

$$
\left[\partial_{z}-Q, \partial_{t}-X\right]=0,
$$

let $\operatorname{Ad}\left(\tilde{g}_{ \pm}^{-1}\right)$ act on the both hand sides to obtain,

$$
\begin{gathered}
{\left[\partial_{z}-a_{ \pm}, \partial_{t}-b_{ \pm}\right]=0,} \\
b_{ \pm}=X^{\tilde{g}_{ \pm}}=\operatorname{Ad}\left(\tilde{g}_{ \pm}^{-1}\right) X+\partial_{t} \tilde{g}_{ \pm}^{-1} \tilde{g}_{ \pm} .
\end{gathered}
$$

Lemma 3.6 says $b_{ \pm} \in \overline{\mathfrak{s}}$, and therefore (3.11) means

$$
\partial_{t} a_{ \pm}=\partial_{z} b_{ \pm} \cdot
$$

This shows that $a_{ \pm}$(or $a_{ \pm, k}$ ) is the conserved current of the system.

2. Reviews of the Toda Lattice Field Theories of Olive and Turok (II). In this section $a_{ \pm}(z, t)$ obtained in the previous section is shown to be a generating function of mutually commuting Hamiltonians, provided that $u(z, t)$ is a (classical) free boson field. The equation of motion with Hamiltonian density $a_{ \pm, k}$ is written in the form of 0-curvature condition between $Q$ and a certain gauge potential $B_{k}$. We will also see that $B_{k}$ 's satisfy 0 -curvature conditions mutually, and this 0 -curvature conditions = integrability condition allows us to introduce a non-local element $c_{ \pm}, \omega_{ \pm}$of gauge groups, which simplify (3.7) and (3.5), as well as connect this theory to the TL hierarchy. Again we closely follow the argument of Olive and Turok $[\mathrm{O}-\mathrm{T} 2,3]$, but avoid using $\mathrm{Tr}$, so that representation independence is obvious.

Suppose there is the local simultaneous canonical commutation relation between $u(z, t)$ and $v(z, t)=\partial u / \partial t$ :

$$
\left\{u_{a}(z, t), v_{b}\left(z^{\prime}, t\right)\right\}=\delta_{a b} \delta\left(z-z^{\prime}\right),
$$

where subscripts $a, b$ specify orthogonal components of $\bar{h}$ with respect to $(\cdot \mid \cdot)$. (For exact meaning of the Poisson bracket $\{$,$\} we refer to [G-Ch], [G-D].)$

Then the fundamental Poisson relation:

$$
\begin{aligned}
& \left\{Q(z, t ; \lambda) \otimes Q\left(z^{\prime}, t ; \mu\right)\right\} \\
& \quad=\left[Q(z, t ; \lambda) \otimes 1+1 \otimes Q\left(z^{\prime}, t ; \mu\right), r(\lambda / \mu)\right] \delta\left(z-z^{\prime}\right) \in \overline{\mathfrak{g}} \otimes \overline{\mathfrak{g}}=\left(\bigoplus_{j \in \mathbf{Z}} \lambda^{j} \dot{\mathfrak{g}}_{j}\right) \otimes\left(\bigoplus_{j \in \mathbf{Z}} \mu^{j} \stackrel{\circ}{\mathfrak{g}}_{j}\right),
\end{aligned}
$$

holds with the classical $r$ matrix $r=r_{ \pm}$:

$$
r_{+}=-\frac{t_{0}}{2}-\sum_{j=1}^{\infty} t_{j}\left(\frac{\lambda}{\mu}\right)^{j}, \quad r_{-}=\frac{t_{0}}{2}+\sum_{j=1}^{\infty} t_{-j}\left(\frac{\mu}{\lambda}\right)^{j} .
$$


Here $t_{j}$ is $\stackrel{\circ}{\mathrm{g}}_{j} \otimes \stackrel{\mathrm{g}}{-j}_{-j}$ component of $i=$ (canonical tensor corresponding to $(\cdot \mid \cdot)$ of $\stackrel{\mathrm{g}}{\text { ) }}$. Belavin and Drinfel'd [B-D] proved that these matrices satisfy the classical Yang-Baxter equation:

$$
\left[r_{12}, r_{13}\right]+\left[r_{12}, r_{23}\right]+\left[r_{13}, r_{23}\right]=0 .
$$

In order to give the equations of motion in the form of 0 -curvature condition, we proceed in two steps. First we prove

Proposition 3.7. ([O-T2]). Set

$$
\begin{aligned}
L_{n} & :=\operatorname{Ad}\left(\tilde{g}_{+}\right) \Lambda_{n}, \quad B_{n}:=\left(1 \otimes L_{n} \mid-r_{+}\right)_{2}, \\
L_{-n} & :=\operatorname{Ad}\left(\tilde{g}_{-}\right) \Lambda_{-n}, \quad B_{-n}:=\left(1 \otimes L_{-n} \mid r_{-}\right)_{2},
\end{aligned}
$$

for $n \in E_{+} .(\cdot \cdot)_{2}$ indicates taking $(\cdot \cdot)$ of the second entries. Then $B_{n}$ is such a local gauge potential that

$$
\begin{aligned}
\left\{Q, \int_{-L}^{L}-a_{+,-n}(z) d z\right\} & =-\frac{\partial B_{n}}{\partial z}+\left[Q, B_{n}\right], \\
\left\{Q, \int_{-L}^{L} a_{-, n}(z) d z\right\} & =-\frac{\partial B_{-n}}{\partial z}+\left[Q, B_{-n}\right] .
\end{aligned}
$$

Proof. Again, for the moment, we are concerned with only $n \in E_{+}$and $a_{+}$. Encapsulating the left side of (3.15) into

$$
\left\{Q \otimes \int_{-L}^{L}-a_{+}(z) d z\right\}
$$

we calculate this, making use of the transition matrix obtained in Sect. 3.1.

According to the abelian property of $a_{+}$,

$$
\begin{aligned}
\left\{Q \otimes \int_{-L}^{L} a_{+}(z) d z\right\} & =\left\{Q \otimes \mathbf{T}_{+, L}\right\}\left(1 \otimes \mathbf{T}_{+, L}^{-1}\right) \\
& =\left(1 \otimes \mathbf{T}_{+}(L, z)^{-1}\right)\left\{Q \otimes \mathbf{T}_{+, L}\right\}\left(1 \otimes \mathbf{T}_{+}(z,-L)^{-1}\right)
\end{aligned}
$$

As $Q$ and $\tilde{g}_{+}$are local,

$$
\left\{Q(z, t) \otimes \tilde{g}_{+}( \pm L, t)\right\}=0 .
$$

This with the explicit form of $\mathbf{T}$ (3.7) yields

$$
\left\{Q \otimes, \mathbf{T}_{+, L}\right\}=\left(1 \otimes \tilde{g}_{+}(L, t)^{-1}\right)\left\{Q \otimes, \mathbf{T}_{L}\right\}\left(1 \otimes \tilde{g}_{+}(-L, t)\right) .
$$

The following important lemma is a consequence of (3.1), and due to the Leningrad school.

Lemma 3.8. (Continuous Leibniz rule; $[\mathrm{Fa}],[\mathrm{Sk}]$ )

$$
\left\{Q(z) \otimes \mathbf{T}_{L}\right\}=\int_{-L}^{L}(1 \otimes \mathbf{T}(L, w))\{Q(z) \underset{,}{Q} Q(w)\}(1 \otimes \mathbf{T}(w,-L)) d w .
$$

Thanks to the fundamental Poisson relation (3.12) and (3.1), the right-hand 
side of this lemma is rewritten as follows:

$$
\begin{aligned}
= & (1 \otimes \mathbf{T}(L, z))[Q(z) \otimes 1+1 \otimes Q(z), r](1 \otimes \mathbf{T}(z,-L)) \\
= & {[Q(z) \otimes 1,(1 \otimes \mathbf{T}(L, z)) r(1 \otimes \mathbf{T}(z,-L))] } \\
& -\frac{\partial}{\partial z}(1 \otimes \mathbf{T}(L, z)) r(1 \otimes \mathbf{T}(z,-L)) .
\end{aligned}
$$

From (3.5), (3.16), (3.17), Lemma 3.8 and (3.18), we get

$$
\left\{Q \otimes \int_{-L}^{L} a_{+}(z) d z\right\}=\left[Q(z) \otimes 1, K_{+}\right]-\frac{\partial K_{+}}{\partial z}+\left[1 \otimes a_{+}(z), K_{+}\right]
$$

where

$$
K_{+}:=\left(1 \otimes \operatorname{Ad}\left(\tilde{g}_{+}(z, t)\right)^{-1}\right) r .
$$

As $\tilde{g}_{+} \in H N_{-}, K_{+}$is well defined for $r=r_{+}$.

In order to extract $\left\{Q, \int_{-L}^{L}-a_{+,-n}(z) d z\right\}$, i.e. $\overline{\mathfrak{g}} \otimes \Lambda_{-n}$ component of (3.19) $\left(n \in E_{+}\right)$, one only needs to take $\left(\cdot \mid 1 \otimes \Lambda_{n}\right)_{2}=\left(\cdot \mid \Lambda_{n}\right)$ of the second entry of both hand sides. Since $\overline{\mathfrak{s}}$ is abelian,

$$
\left(\left[a_{+}(z), x\right] \mid \Lambda_{n}\right)=\left(x \mid\left[\Lambda_{n}, a_{+}(z)\right]\right)=0 .
$$

Hence the last term of (3.19) does not contribute to the final result. The invariant property also yields

$$
\left(1 \otimes \Lambda_{n} \mid K_{+}\right)_{2}=\left(1 \otimes L_{n} \mid r_{+}\right)_{2}=-B_{n},
$$

and substituting this into (3.19), we obtain (3.15).

As the second step, so as to interpret (3.15) as 0-curvature condition, we check the compatibility;

\section{Proposition 3.9.}

$$
H_{ \pm n}:=\int_{-L}^{L} \mp a_{ \pm, \mp n}(z) d z \quad\left(n \in E_{+}\right)
$$

are mutually commuting with respect to $\{$,$\} .$

Proof. Similar to that of the previous proposition.

$\left\{H_{k}, H_{n}\right\}=0\left(k \in E_{+}, n \in E_{ \pm}\right)$is $\Lambda_{-k} \otimes \Lambda_{-n}$ component of

$$
\left\{\int_{-L}^{L} a_{+}(z) d z, H_{n}\right\}=0
$$

Now we prove (3.21). Just like we get (3.17), the left-hand side of (3.21) is

$$
\begin{aligned}
\left\{\int_{-L}^{L} a_{+}(z) d z, H_{n}\right\}= & -\tilde{g}_{+}(L, t)^{-1}\left\{\tilde{g}_{+}(L, t), H_{n}\right\}+\mathbf{T}_{+. L} \tilde{g}_{+}(L, t)^{-1}\left\{\tilde{g}_{+}(L, t), H_{n}\right\} \mathbf{T}_{+, L}^{-1} \\
& +\tilde{g}_{+}(L, t)^{-1}\left\{\mathbf{T}_{L}, H_{n}\right\} \tilde{g}_{+}(L, t) \mathbf{T}_{+. L}^{-1} .
\end{aligned}
$$


By Lemma 3.8, (3.15) and (3.1), it is easy to see

$$
\left\{\mathbf{T}_{L}, H_{n}\right\}=-B_{n} \mathbf{T}_{L}+\mathbf{T}_{L} B_{n}
$$

Replacing (3.23) into (3.22), we obtain

$$
\left\{\int_{-L}^{L} a_{+}(z) d z, H_{n}\right\}=-C+\operatorname{Ad}\left(\mathbf{T}_{+, L}\right) C,
$$

where

$$
C=\tilde{g}_{+}(L, t)^{-1}\left\{\tilde{g}_{+}(L, t), H_{n}\right\}+\tilde{g}_{+}(L, t)^{-1} B_{n} \tilde{g}_{+}(L, t) .
$$

As $\mathbf{T}_{+, L} \in \exp (\overline{\mathfrak{s}})$, the same argument as (3.20) shows that (3.24) implies (3.21).

Taking this proposition into account, we introduce new independent variables $x_{n}\left(n \in E_{ \pm}\right)$conjugate to the Hamiltonian $H_{n}$. Thus (3.15) becomes the 0 -curvature condition

$$
\left[\partial_{z}-Q, \partial_{x_{n}}-B_{n}\right]=0
$$

as was expected.

Example. Honestly following the proof of Theorem 3.3, we obtain

$$
\begin{gathered}
B_{1}=\frac{\partial_{t} u+\partial_{z} u}{4}+e^{\mathrm{ad} u / 2} \Lambda_{+}, \\
B_{-1}=\frac{\partial_{t} u-\partial_{z} u}{4}+e^{-\mathrm{ad} u / 2} \Lambda_{-},
\end{gathered}
$$

that is,

$$
B_{1}+B_{-1}=Q, \quad B_{1}-B_{-1}=X .
$$

Hence we may identify $z$ and $t$ as

$$
z=\frac{1}{2}\left(x_{1}+x_{-1}\right), \quad t=\frac{1}{2}\left(x_{1}-x_{-1}\right) .
$$

Now we study the gauge potentials $B_{n}\left(n \in E_{+} \cup E_{-}\right)$in detail, following [O-T3].

Because of the fact $\tilde{g}_{ \pm} \in H N_{\mp}$ and the explicit form of $r=r_{ \pm}(3.13)$,

$$
\begin{array}{cc}
L_{n} \in \bigoplus_{k \leqq n} \overline{\mathfrak{g}}_{k}, & B_{n} \in \bigoplus_{k=0}^{n} \overline{\mathfrak{g}}_{k}, \\
L_{-n} \in \bigoplus_{k \geqq-n} \overline{\mathfrak{g}}_{k}, & B_{-n} \in \bigoplus_{k=0}^{n} \overline{\mathfrak{g}}_{-k},
\end{array}
$$

for $n \in E_{+}$, and

$$
\left(B_{n}\right)_{k}= \begin{cases}\left(L_{n}\right)_{k}, & k>0(\text { respectively } k<0) \\ \frac{1}{2}\left(L_{n}\right)_{0}, & k=0(\text { respectively } k=0) \\ 0, & k<0(\text { respectively } k>0)\end{cases}
$$

for $n \in E_{+}$(respectively $n \in E_{-}$). Here $(x)_{k}$ is the $\overline{\mathfrak{g}}_{k}$ component of $x$. 
Theorem 3.10. For all $n, m \in E_{+} \cup E_{-}, B_{n}$ and $L_{m}$ satisfy the Lax equation:

$$
\left[\partial_{x_{n}}-B_{n}, L_{m}\right]=0 \text {. }
$$

Proof. Fix $n \in E_{+}$. Then

$$
\left(B_{n}\right)^{\tilde{g}_{+}} \in \bigoplus_{k \leqq n} \overline{\mathfrak{g}}_{k}, \quad\left(B_{n}\right)^{\tilde{g}_{-}} \in \bigoplus_{k \geqq 0} \overline{\mathfrak{g}}_{k},
$$

and, applying $\operatorname{Ad}\left(\tilde{g}_{ \pm}\right)^{-1}$ to (3.25), we obtain

$$
\left[\partial_{z}-a_{ \pm}, \partial_{x_{n}}-\left(B_{n}\right)^{\tilde{g}_{ \pm}}\right]=0 .
$$

So the assumption of Lemma 3.6 are satisfied. Hence $\left(B_{n}\right)^{\tilde{g}_{ \pm} \in \overline{\mathfrak{s}}}$ and

$$
\left[\partial_{x_{n}}-\left(B_{n}\right)^{\tilde{g}_{ \pm}}, \Lambda_{m}\right]=0
$$

for any exponent $m$. Applying $\operatorname{Ad}\left(\tilde{g}_{ \pm}\right),(3.29)$ is proved. The case $n \in E_{-}$is similar.

Olive and Turok [O-T3] derive the 0-curvature condition:

$$
\left[\partial_{x_{n}}-B_{n}, \partial_{x_{m}}-B_{m}\right]=0, \quad n, m \in E_{+} \cup E_{-},
$$

from Lax equations (3.29) by making use of the classical Yang-Baxter equation. But, noting that $B_{n}$ is the "upper (or lower) half" of $L_{n}(3.28)$, exactly the same argument as the proof of Proposition 1.1 stands to prove (3.30) more easily. This fact may suggest some interpretation of the role of the classical $r$ matrix and the classical Yang-Baxter equation in the theory of integrable systems.

Remark 3.11. Under the identification (3.26) the original Toda equation is recovered as (3.30) $n=1, m=-1$.

We now introduce non-local elements $\omega_{ \pm}$of gauge groups, which behave just like wave matrices $\hat{W}_{\infty}^{(0)}$ of the TL hierarchy. As shown in the proof of Theorem 3.10,

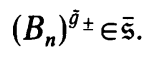

Along with (3.28), it implies

$$
\begin{aligned}
& C_{n}^{+}:=\left(B_{n}\right)^{\tilde{g}_{+}-\Lambda_{n} \in \overline{\mathfrak{s}}_{-},} \text {for } n \in E_{+}, \\
& C_{n}^{-}:=\left(B_{n}\right)^{\tilde{g}_{-}} \quad \in \overline{\mathfrak{s}}_{+}, \\
& C_{n}^{+}:=\left(B_{n}\right)^{\tilde{g}_{+}} \quad \in \overline{\mathfrak{s}}_{-}, \quad \text { for } n \in E_{-}, \\
& C_{n}^{-}:=\left(B_{n}\right)^{\tilde{g}_{-}-\Lambda_{n} \in \overline{\mathfrak{s}}_{+},}
\end{aligned}
$$

where

$$
\begin{aligned}
& \overline{\mathfrak{s}}_{+}=\bigoplus_{j \in E_{+}} \overline{\mathfrak{s}}_{j}, \\
& \overline{\mathfrak{s}}_{-}=\bigoplus_{j \in E_{-}} \overline{\mathfrak{s}}_{j} .
\end{aligned}
$$

(3.30) is equivalent to

$$
\left[\partial_{x_{n}}-\left(B_{n}\right)^{\tilde{g}_{ \pm}}, \partial_{x_{m}}-\left(B_{m}\right)^{\tilde{g}_{ \pm}}\right]=0,
$$


therefore also equivalent to

$$
\begin{aligned}
& {\left[\partial_{x_{n}}-C_{n}^{+}, \partial_{x_{m}}-C_{m}^{+}\right]=\partial_{x_{m}} C_{n}^{+}-\partial_{x_{n}} C_{m}^{+}=0,} \\
& {\left[\partial_{x_{n}}-C_{n}^{-}, \partial_{x_{m}}-C_{m}^{-}\right]=\partial_{x_{m}} C_{n}^{-}-\partial_{x_{n}} C_{m}^{-}=0 .}
\end{aligned}
$$

They describe the integrability condition of the linear problems for $C^{+} \in \overline{\mathfrak{s}}_{-}$and

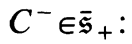

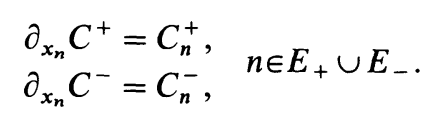

Gauge transformation by $c^{ \pm}:=\exp C^{ \pm} \in N_{\mp}$ yields from (3.31),

$$
\left.\left.\begin{array}{l}
\left(B_{n}\right)^{\tilde{g}_{+} c_{+}}=\Lambda_{n} \\
\left(B_{n}\right)^{\tilde{g}_{-} c_{-}}=0
\end{array}\right\} \text { for } \quad n \in E_{+}, \begin{array}{l}
\left(B_{n}\right)^{\tilde{g}_{+} c_{+}}=0 \\
\left(B_{n}\right)^{\tilde{g}_{-} c_{-}}=\Lambda_{n}
\end{array}\right\} \text { for } n \in E_{-}
$$

Setting $\omega_{ \pm}:=\tilde{g}_{ \pm} c_{ \pm}=h_{ \pm} g_{ \pm} c_{ \pm}$, we obtain

Theorem 3.12.

$$
\begin{aligned}
\partial_{x_{ \pm n}} \omega_{ \pm} & =B_{ \pm n} \omega_{ \pm}-\omega_{ \pm} \Lambda_{ \pm n}, \\
\partial_{x_{ \pm n}} \omega_{\mp} & =B_{ \pm n} \omega_{\mp}, \\
L_{ \pm n} & =\operatorname{Ad}\left(\omega_{ \pm}\right) \Lambda_{ \pm n},
\end{aligned}
$$

for $n \in E_{+}$.

Remark 3.13. $C^{ \pm}$is determined up to an additive constant which is the initial value of the system (3.33). Hence $\omega_{ \pm}$is unique up to a constant multiplier in exp $\bar{s}$.

Corollary 3.14.

$$
\begin{aligned}
\mathbf{T}\left(z^{1}, z^{2}\right) & =\omega_{ \pm}\left(z^{1}\right) \exp \left(z^{1}-z^{2}\right) \Lambda_{ \pm} \omega_{ \pm}\left(z^{2}\right)^{-1} \\
\mathbf{T}_{ \pm}\left(z^{1}, z^{2}\right) & =c_{ \pm}\left(z^{1}\right) \exp \left(z^{1}-z^{2}\right) \Lambda_{ \pm} c_{ \pm}\left(z^{2}\right)^{-1}
\end{aligned}
$$

Proof. It suffices to check the defining equations of $\mathbf{T}, \mathbf{T}_{ \pm}$, i.e. (3.1) and (3.6), using the identifications (3.26) and Theorem 3.12.

3. Generalized TL Hierarchy. The analogy of the TL hierarchy (Sect. 1) and the system studied in Sects. 3.1, 2 is now obvious. Observing (1.1) $\alpha=0$ and (3.28), (1.3L) and (3.29), (1.3ZS) and (3.30), (1.2) and Theorem 3.12, we present here a candidate for the generalized TL hierarchy attached to the affine Lie algebra which includes the system of Olive and Turok (Sect. $3.2(3.29)$ and (3.30)) as a special case.

Let $h_{0}(x) \in H, \hat{W}_{0}^{(0)}(x) \in N_{+}, \hat{W}_{0}^{(\infty)}(x) \in N_{-}$be unknown functions depending on $x=\left(x_{n} ; n \in E_{+} \cup E_{-}\right)$, and set

$$
\begin{array}{lll}
L_{n}:=\operatorname{Ad}\left(h_{0} \hat{W}_{0}^{(\infty)}\right) \Lambda_{n} . & B_{n}:=\left(L_{n}\right)_{+}+\frac{1}{2}\left(L_{n}\right)_{0}, & n \in E_{+}, \\
L_{n}:=\operatorname{Ad}\left(h_{0}^{-1} \hat{W}_{0}^{(0)}\right) \Lambda_{n}, & B_{n}:=\left(L_{n}\right)_{-}+\frac{1}{2}\left(L_{n}\right)_{0}, & n \in E_{-} .
\end{array}
$$

Here ()$_{ \pm, 0}$ means

$$
(x)_{+}:=\sum_{k>0} x_{k}, \quad(x)_{-}:=\sum_{k<0} x_{k}, \quad(x)_{0}:=x_{0}
$$


for $x=\sum x_{k}, x_{k} \in \overline{\mathfrak{g}}_{k}$. We call the following system the generalized TL hierarchy associated to the affine Lie algebra $\mathrm{g}$ :

$$
\partial_{x_{n}} L_{m}=\left[B_{n}, L_{m}\right]
$$

for the exponents $n, m$, or equivalently

$$
\left[\partial_{x_{n}}-B_{n}, \partial_{x_{m}}-B_{m}\right]=0 .
$$

Equivalence of (3.35L) and (3.35ZS) is proved similarly to Proposition 1.1 of Sect. 1, and parallel to Theorem 3.12 there exist wave matrices of the form

$$
\begin{aligned}
W^{(\infty)}(x) & =h_{+}(x) \hat{W}^{(\infty)}(x) \exp \xi_{\mathrm{g}}\left(x_{+}\right), \\
W^{(0)}(x) & =h_{-}(x) \hat{W}^{(0)}(x) \exp \xi_{\mathrm{g}}\left(x_{-}\right),
\end{aligned}
$$

where

$$
h_{ \pm}(x)=h(x)^{ \pm 1} \in H, \quad \hat{W}^{\left(\begin{array}{l}
0 \\
\infty
\end{array}\right)}(x) \in N_{ \pm},
$$

and $\xi_{g}\left(x_{ \pm}\right)=\sum_{n \in E_{ \pm}} x_{n} \Lambda_{n}$, which are the solution of the linear problem:

$$
\partial_{x_{n}} W^{\left(\begin{array}{c}
0 \\
\infty
\end{array}\right)}(x)=B_{n} W^{\left(\begin{array}{c}
0 \\
\infty
\end{array}\right)}(x)
$$

for the exponent $n$. The characterization of the solution by the bilinear relation

$$
\mathbf{T}:=W^{(\infty)}(x) W^{(\infty)}\left(x^{\prime}\right)^{-1}=W^{(0)}(x) W^{(0)}\left(x^{\prime}\right)^{-1},
$$

is also the same as the TL hierarchy of Ueno and Takasaki [U-T].

An important ingredient of Sato-type theory for integrable systems still lacking here is the theory of generalized $\tau$ functions. This will be treated in the forthcoming paper. A promising suggestion is found in [Ch2], and it must be closely related to (and probably included in) the theory of generalized hierarchy [K-W]. The intimate relations between the TL hierarchy and the KP hierarchy must inherit in the above system, and should also be further studied (cf. Sect. 4)

4. The Case $\mathrm{g}=A_{l-1}^{(1)}$. In this section, apart from the general theory, we restrict ourselves to the case $\mathrm{g}=A_{l-1}^{(1)}$ and investigate the relation of the TL hierarchy and Hamiltonians in detail. When $g$ is represented as infinite matrices, the generalized TL hierarchy presented in the previous section is shown to be the $l$-reduced TL hierarchy of [U-T], and the Hamiltonian obtained in Sect. 3.2 is expressed in terms of the $\tau$ function. We keep the notations of the previous section.

It is well known $[\mathrm{Ka}][\mathrm{K}-\mathrm{R}]$ that $\overline{\mathrm{g}}=\overline{A_{l-1}^{(1)}}$ has a faithful representation as $\mathbf{Z} \times \mathbf{Z}$ matrices, such that $A \in \overline{\mathfrak{g}}$ is represented as

$$
A=\left(\begin{array}{ccccc}
\ddots & \ddots & \ddots & & \\
\ddots & A_{0} & A_{1} & A_{2} & \\
\ddots & A_{-1} & A_{0} & A_{1} & \ddots \\
& A_{-2} & A_{-1} & A_{0} & \ddots \\
& & \ddots & \ddots & \ddots
\end{array}\right)=\sum_{i \in \mathbb{Z}} A_{i} \otimes \Lambda^{i}, \quad A_{i} \in \mathfrak{s l}(l, \mathbf{C}),
$$

where $A_{i}=0$ for all but finite $i$. Exponents of $\mathfrak{g}$ are integers $\not \equiv 0\left(\bmod h^{(\kappa)}=l\right)$. 

The principal subalgebra is $\overline{\mathfrak{s}}=\underset{n \neq 0(\bmod l)}{C_{n}} \mathrm{C} \Lambda^{n}, \Lambda_{ \pm}=\Lambda^{ \pm 1}$. ( $\Lambda$ is the shift matrix as
in Sect. 1.)

Theorem 3.15. The solution of the generalized TL hierarchy associated to $A_{l-1}^{(1)}$ in this representation gives the solution of the l-reduced TL hierarchy ( $\alpha=0$ gauge) and vice versa in natural way. That is, they are considered identical with each other.

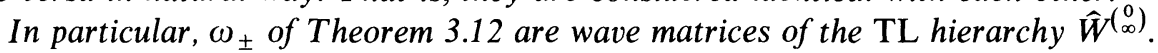

Proof. Suppose the solution of the generalized TL hierarchy is given. Then in the above representation $L_{n}=\left(L_{1}\right)^{n}$ and $L_{-n}=\left(L_{-1}\right)^{n}$ for $n \in E_{+}$, and $B_{n}$ 's are identical with those determined by (1.1) $\alpha=0$. We consider them as dependent on $x_{n}$ $(n \equiv 0(\bmod l))$ trivially. Since they satisfy $(3.35)$, they are solutions of the TL hierarchy. Moreover, as $h_{0}$ and $\hat{W}^{\left(\begin{array}{c}0 \\ \infty\end{array}\right)}$ are $l$-periodic matrices, $\left(L_{ \pm 1}\right)^{l}=\Lambda^{ \pm l}$. Therefore we obtain the solution of the $l$-periodic reduced TL hierarchy.

Conversely, suppose the solution of the $l$-reduced TL hierarchy is given. Using the loop algebra representation of periodic matrix algebra, it is easy to see that $\left(L_{ \pm}\right)^{n}$ and $B_{ \pm n}$ are in $A_{l-1}^{(1)}$ (cf. [U-T] Proposition $1.15(2)$ ). Let $\hat{W}^{\left(\begin{array}{l}0 \\ \infty\end{array}\right)}(x)$ be wave matrices and set

$$
h_{0}:=e^{-u / 2}, \quad \tilde{W}^{\left(\begin{array}{c}
0 \\
\infty
\end{array}\right)}:=h_{0}^{ \pm 1} \hat{W}_{\left(\begin{array}{c}
0 \\
\infty
\end{array}\right)}^{(x)},
$$

where $u=\operatorname{diag}[u(s)]$. $\tilde{W}^{(\infty)}$ (respectively $\tilde{W}^{(0)}$ ) is an $l$-periodic and lower (respectively upper) triangular matrix with the diagonal part 1 . Hence

$$
\begin{aligned}
\tilde{X}^{(\infty)} & =\sum_{k<0} \tilde{X}_{k}^{(\infty)} \otimes \Lambda^{k}:=\log \tilde{W}^{(\infty)}, \\
\left(\text { respectively } \tilde{X}^{(0)}\right. & \left.=\sum_{k>0} \tilde{X}_{k}^{(0)} \otimes \Lambda^{k}:=\log \tilde{W}^{(0)}\right)
\end{aligned}
$$

is also $l$-periodic and strictly lower (respectively upper) triangular, though, in general, $\tilde{X}_{k}^{\left(\begin{array}{c}0 \\ (\end{array}\right)} \in \operatorname{gl}(l, \mathbf{C})$ and $\tilde{X}_{(\infty)}^{\left(\begin{array}{c}0 \\ \infty\end{array}\right)}$ may not belong to $\overline{\mathfrak{g}}$. But subtracting the trace part

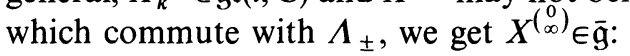

$$
X^{\left(\begin{array}{c}
0 \\
\infty
\end{array}\right)}=\sum\left(\tilde{X}_{k}^{\left(\begin{array}{c}
0 \\
\infty
\end{array}\right)}-\operatorname{Tr} \tilde{X}_{k}^{\left(\begin{array}{c}
0 \\
(\infty)
\end{array}\right)} \operatorname{Id}_{l}\right) \otimes \Lambda^{k}
$$

and $\hat{W}_{0}^{\left(\begin{array}{l}0 \\ \infty\end{array}\right)}:=\exp X^{\left(\begin{array}{l}0 \\ \infty\end{array}\right)}$ gives the same $L_{ \pm}$as $\hat{W}^{\left(\begin{array}{c}0 \\ \infty\end{array}\right)}$ :

$$
L_{+}=\operatorname{Ad}\left(h_{0} \hat{W}^{(\infty)}\right) \Lambda_{+}, \quad L_{-}=\operatorname{Ad}\left(h_{0}^{-1} \hat{W}_{0}^{(0)}\right) \Lambda_{-} .
$$

Clearly, $\left(L_{ \pm}\right)^{n}$ and $B_{ \pm n}$ of the TL hierarchy satisfy the algebraic constraints for $L_{n}$ and $B_{ \pm n}(3.34)$, and (1.3) says that they satisfy (3.35).

The last statement is obvious from Theorem 3.12 .

Especially, the Hamiltonian system constructed in Sect. 3.2 is one of solutions of the TL hierarchy.

As the TL hierarchy is entirely described by the $\tau$ function, Hamiltonian densities of the system of Sect. 3.2 should also be expressed in terms of the $\tau$ function.

Theorem 3.16. In the notations of Sect. 3.2,

$$
H_{n}=-\int_{-L}^{L} p_{n}\left(-\tilde{\partial}_{x_{+}}\right) \partial_{z} \log \tau d z
$$




$$
H_{-n}=\int_{-L}^{L} p_{n}\left(-\tilde{\partial}_{x_{-}}\right) \partial_{z} \log \tau d z .
$$

For the definition of $p_{j}$, see Sect. 1.1.

Proof. Set in the matrix representation

$$
\begin{aligned}
& \tilde{g}_{ \pm}=h_{ \pm} g_{ \pm}=\sum_{j=0}^{\infty} \operatorname{diag}\left[\tilde{g}_{ \pm j}(s)\right] \Lambda^{ \pm j}, \\
& c_{ \pm}=\sum_{j=0}^{\infty} c_{ \pm j} \Lambda^{\mp j}, \quad C^{ \pm}=\sum_{j=0}^{\infty} C_{j}^{ \pm} \Lambda^{\mp j}, \\
& \omega_{ \pm}=\sum_{j=0}^{\infty} \operatorname{diag}\left[\omega_{ \pm j}(s)\right] \Lambda^{\mp j},
\end{aligned}
$$

and with an indeterminant $\lambda$

$$
\begin{aligned}
\tilde{g}_{ \pm}(s ; \lambda) & =\sum_{j=0}^{\infty} \operatorname{diag}\left[\tilde{g}_{ \pm j}(s)\right] \lambda^{\mp j} \\
c_{ \pm}(\lambda) & =\sum_{j=0}^{\infty} c_{ \pm j} \lambda^{\mp j}, \quad C^{ \pm}(\lambda)=\sum_{j=0}^{\infty} C^{ \pm}{ }_{j} \lambda^{\mp j} \\
\omega_{ \pm}(s ; \lambda) & =\sum_{j=0}^{\infty} \operatorname{diag}\left[\omega_{ \pm j}(s)\right] \lambda^{\mp j} .
\end{aligned}
$$

( $\lambda$ is identical with that in principal realization of $g$.) Then, as $\omega_{ \pm}=\tilde{g}_{ \pm} c_{ \pm}$and $c_{ \pm}=\exp \left(C^{ \pm}\right)$

$$
\omega_{ \pm}(s ; \lambda)=\tilde{g}_{ \pm}(s ; \lambda) c_{ \pm}(\lambda), \quad c_{ \pm}(\lambda)=\exp \left(C^{ \pm}(\lambda)\right) .
$$

Hence

$$
C^{ \pm}(\lambda)=\log \omega_{ \pm}(s ; \lambda)-\log \tilde{g}_{ \pm}(s ; \lambda)
$$

Equations (3.33) and (3.26) imply $\partial_{z} C^{ \pm}{ }_{j}=a_{ \pm, \mp j}$, i.e.

$$
\mp \sum_{j=0}^{\infty} H_{ \pm j} \lambda^{\mp j}=\int_{-L}^{L} \partial_{z} C^{ \pm}(\lambda) d z
$$

Substituting (3.40) into (3.41), we obtain

$$
\mp \sum_{j=0}^{\infty} H_{ \pm j} \lambda^{\mp j}=\int_{-L}^{L} \partial_{z} \log \omega_{ \pm}(s ; \lambda) d z-\int_{-L}^{L} \partial_{z} \log \tilde{g}_{ \pm}(s ; \lambda) d z .
$$

As $\tilde{g}$ is local, the last term vanishes because of the periodicity in $z$. On the other hand, being wave matrices of the TL hierarchy, $\omega_{ \pm}$is described by the $\tau$ function (cf. Theorem $1.7[\mathrm{U}-\mathrm{T}]$ ):

$$
\begin{aligned}
& \log \omega_{+}(s ; \lambda)=-\frac{1}{2} u(s ; x)+\sum_{j=1}^{\infty} p_{j}\left(-\tilde{\partial}_{x_{+}}\right) \log \tau(s ; x) \lambda^{-j}, \\
& \log \omega_{-}(s ; \lambda)=\frac{1}{2} u(s ; x)+\sum_{j=1}^{\infty} p_{j}\left(-\tilde{\partial}_{x_{-}}\right) \log \tau(s ; x) \lambda^{j}
\end{aligned}
$$

Equations (3.42) and (3.43) prove the theorem. 
The fact that $\log \omega_{ \pm}(s ; \lambda)=\log \hat{w}^{\left(\begin{array}{c}0 \\ \infty\end{array}\right)}(s ; \lambda)$ is generating functions of conserved quantities, will be derived from completely different point of view in Sect. 4 .

\section{Conservation Laws for the Multi-Component KP Hierarchy}

In this section, we extend the theory of conservation laws for the KP hierarchy ( $[\mathrm{Ch} 1, \mathrm{~W}, \mathrm{~F} 1 ; \mathrm{Sa}])$ to the multi-component $\mathrm{KP}$ hierarchy. In particular conservation laws for the 2-component KP hierarchy include the Hamiltonian densities of the TL hierarchy embedded in it.

1. Reviews of the Multi-Component KP Hierarchy. First we recall the multicomponent KP hierarchy briefly, following the Appendix 1.3 [U-T].

The independent variables of the $r$ component theory are $x=\left(x^{(1)}, \ldots, x^{(r)}\right)$, $x^{(\alpha)}=\left(x_{1}^{(\alpha)}, x_{2}^{(\alpha)}, \ldots\right)$, and functions concerned belong to $\mathscr{A}$, a suitable differential algebra of $r \times r$ matrix valued functions of $x$ with the derivation

$$
\partial=\sum_{\alpha=1}^{r} \partial_{x_{1}^{(\alpha)}}
$$

Let $L, U_{\alpha}(\alpha=1, \ldots, r)$ be the following micro-differential operators:

$$
\begin{aligned}
L & =\sum_{j \leqq 1} u_{j} \partial^{j}, \quad \text { with } u_{j} \in \mathscr{A}, \quad u_{1}=\mathrm{Id}_{r}, u_{0}=0, \\
U_{\alpha} & =\sum_{j \leqq 0} u_{j, \alpha} \partial^{j}, \quad \text { with } \quad u_{j, \alpha} \in \mathscr{A}, u_{0}=E_{\alpha} .
\end{aligned}
$$

$E_{\alpha}$ is the matrix element $\left(\delta_{i \alpha} \delta_{j \alpha}\right)_{1 \leqq i, j \leqq r}$. We assume that $L$ and $U_{\alpha}$ 's satisfy the following algebraic conditions:

$$
\begin{aligned}
& {\left[L, \mathrm{U}_{\alpha}\right]=0, \quad\left[U_{\alpha}, U_{\beta}\right]=0} \\
& \sum_{\alpha=1}^{r} U_{\alpha}=\mathrm{Id}_{r}, \quad U_{\alpha} U_{\beta}=\delta_{\alpha \beta} U_{\alpha}
\end{aligned}
$$

for $\alpha, \beta=1, \ldots, r$. Set

$$
B_{n}^{(\alpha)}:=\left(L^{n} U_{\alpha}\right)_{+},
$$

where $P_{+}$means the differential operator part of a micro-differential operator $P$. The $r$ component KP hierarchy is, by definition, the compatibility condition of the linear problem,

$$
\begin{gathered}
L W=\lambda W, \quad U_{\alpha} W=W E_{\alpha}, \quad \alpha=1, \ldots, r, n=1,2, \ldots, \\
\partial_{x_{n}^{(\alpha)}} W=B_{n}^{(\alpha)} W,
\end{gathered}
$$

where $\lambda$ is a formal parameter, on which $W$ depends. Explicitly, the KP hierarchy is the following system of unknown functions $u_{j}, u_{j, \alpha}$ for all $\alpha=1, \ldots, r, n=1,2, \ldots$ :

$$
\partial_{x_{n}^{(\alpha)}} L=\left[B_{n}^{(\alpha)}, L\right], \quad \partial_{x_{n}^{(\alpha)}} U_{\beta}=\left[B_{n}^{(\alpha)}, U_{\beta}\right]
$$

or equivalently

$$
\left[\partial_{x_{n}^{(\alpha)}}-B_{n}^{(\alpha)}, \partial_{x_{m}^{(\beta)}}-B_{m}^{(\beta)}\right]=0
$$


Suppose $L$ and $U_{\alpha}$ 's are a solution of the $r$ component KP hierarchy. Then the linear problem (4.4) has a solution of the form:

$$
W(x ; \lambda)=\sum_{j=0}^{\infty} \hat{w}_{j}(x) \lambda^{-j} \exp \left(\sum_{\alpha=1}^{r} \xi\left(x^{(\alpha)}, \lambda\right) E_{\alpha}\right),
$$

with $\hat{w}_{j} \in \mathscr{A}$ and $\hat{w}_{0}=\mathrm{Id}_{r}$. Setting

$$
\hat{W}(x ; \partial):=\sum_{j=0}^{\infty} \hat{w}_{j}(x) \partial^{-j}
$$

we can rewrite (4.4) into

$$
\begin{aligned}
L \hat{W} & =\hat{W} \partial, \quad U_{\alpha} \hat{W}=\hat{W} E_{\alpha}, \\
\partial_{x_{n}^{(\alpha)}} \hat{W} & =B_{n}^{(\alpha)} \hat{W}-\hat{W} E_{\alpha} \partial^{n} .
\end{aligned}
$$

Matrix elements of $\hat{W}(x ; \lambda)$ are written in terms of $\tau$ functions, $\tau$ and $\tau_{\alpha \beta}$ :

$$
\hat{W}(x ; \lambda)_{\alpha \beta}= \begin{cases}\tau\left(x-\varepsilon_{\beta}(\lambda)\right) / \tau(x), & \alpha=\beta, \\ \tau_{\alpha \beta}\left(x-\varepsilon_{\beta}(\lambda)\right) / \tau(x), & \alpha \neq \beta,\end{cases}
$$

where $\varepsilon_{\alpha}(\lambda)=(0, \ldots, 0, \varepsilon(\lambda), 0, \ldots, 0), \varepsilon(\lambda)=\left(\lambda, \lambda^{2} / 2, \lambda^{3} / 3, \ldots\right)$. One of important results of $[\mathrm{U}-\mathrm{T}]$ is the embedding theorem of the TL hierarchy.

Theorem 4.1. ([U-T]). The $\tau$ function of the TL hierarchy $\tau\left(s ; x_{ \pm}\right)$coincides with that of the 2-component KP hierarchy up to a signature factor under the identification of the independent variables $x^{(1)}=x_{+}, x^{(2)}=x_{-}$;

$$
\tau\left(x^{(1)}, x^{(2)}\right)=\tau\left(0 ; x_{ \pm}\right), \quad \tau_{12}\left(x^{(1)}, x^{(2)}\right)=\tau\left(1 ; x_{ \pm}\right) .
$$

For the correspondence between $\tau\left(s ; x_{ \pm}\right), s \neq 0,1$ and $\tau$ functions of the KP hierarchy, see [U-T]. The statement cited above is the minimum of what we need (cf. also [T]).

2. Conservation Laws for the Multi-Component KP Hierarchy. Here we show that the diagonal part (or exactly speaking, logarithm of the diagonal part) of the wave operator $\hat{W}$ gives the conservation laws. The $\tau$ function is shown to be its generating function, and the relation to the Hamiltonian densities of the TL hierarchy is discussed.

As in Sect. 3 we call those functions local, which can be expressed as differential polynomials of coefficients of $L$ and $U_{\alpha}$, i.e. $u_{j}$ and $u_{j, \alpha}$. Suppose there exists a "good" boundary condition for a variable $z=\left(\right.$ a linear combination of $\left.x_{n}^{(\alpha)}\right)$, under which a local quantity is constant on the boundary; for example

- Periodic condition for $L$ and $U_{\alpha}$,

$$
L\left(z+L_{z}\right)=L\left(z-L_{z}\right), \quad U_{\alpha}\left(z+L_{z}\right)=U_{\alpha}\left(z-L_{z}\right) .
$$

- Rapidly decreasing condition at $z= \pm \infty$ for $L$ and $U_{\alpha}$.

Then

$$
\oint \partial_{z}(\text { local quantity }) d z=0
$$


where $\oint$ is the integration over the whole period (i.e. Periodic case: $\int_{-L_{z}}^{L_{z}}$; Rapidly decreasing case: $\left.\int_{-\infty}^{\infty}\right)$. The following proposition is trivial but fundamental.

Proposition 4.2. Let $H(x)$ be a (possibly non-local) function, and suppose $\partial_{x_{n}^{(\alpha)}} H(x)$ is local. Then

$$
\partial_{x_{n}^{(\alpha)}}\left(\oint \partial_{z} H(x) d z\right)=0 .
$$

This means that $\oint \partial_{z} H(x) d z$ is conserved with respect to $x_{n}^{(\alpha)}$.

We define

$$
\eta(x \cdot \lambda)=\left(\begin{array}{ccc}
\eta_{1}(x, \lambda) & & 0 \\
0 & & \eta_{r}(x, \lambda)
\end{array}\right):=\left(\begin{array}{ccc}
\log \hat{W}_{11} & \ddots & 0 \\
0 & & \log \hat{W}_{r r}
\end{array}\right),
$$

and

$$
\eta_{\alpha}(x, \lambda)=\sum_{j=1}^{\infty} H_{\alpha, j}(x) \lambda^{-j} .
$$

Note that $\hat{W}$ is obtained by integrating $L$ and $U_{\alpha}$, and hence $\eta$ and $H_{\alpha, j}$ are not local.

Theorem 4.3. For $\alpha=1, \ldots, r, n=1,2, \ldots$,

$$
\partial_{x_{n}^{(\alpha)}} \eta(x ; \lambda) \text { or, equivalently } \partial_{x_{n}^{(\alpha)}} H_{\alpha, j} \text { is local. }
$$

By Proposition 4.2 this means $\oint \partial_{z} H_{\alpha, j}(x) d z$ is conserved with respect to any $x_{n}^{(\alpha)}$. Proof. First we prove a lemma:

Lemma 4.4. $\hat{W}_{\alpha \beta}(x ; \lambda) / \widehat{W}_{\beta \beta}(x ; \lambda)(\alpha \neq \beta)$ is local.

Proof. From (4.9),

$$
\frac{\hat{W}_{\alpha \beta}}{\hat{W}_{\beta \beta}}=\left.\lambda^{-1} \frac{\tau_{\alpha \beta}\left(x^{\prime}\right)}{\tau\left(x^{\prime}\right)}\right|_{x^{\prime}=x-\varepsilon_{\beta}(\lambda)}=\left.\lambda^{-1}\left(\hat{w}_{1}\left(x^{\prime}\right)\right)_{\alpha \beta}\right|_{x^{\prime}=x-\varepsilon_{\beta}(\lambda)} .
$$

So, we need only to prove that $\left(\hat{w}_{1}(x)\right)_{\alpha \beta}$ is local.

On the other hand, $y_{1, \gamma}$, the coefficient of $\lambda^{-1}$ in $U_{\gamma}(x ; \lambda)=\hat{W} E_{\gamma} \hat{W}^{-1}$, is

$$
\begin{aligned}
u_{1, \gamma} & =\hat{w}_{1}(x) E_{\gamma}-E_{\gamma} \hat{w}_{1}(x), \\
\left(u_{1, \gamma}\right)_{\alpha \beta} & =\left(\hat{w}_{1}(x)\right)_{\alpha \beta}\left(\delta_{\beta \gamma}-\delta_{\alpha \gamma}\right) .
\end{aligned}
$$

Thus $\left(\hat{w}_{1}(x)\right)_{\alpha \beta}$ is local, if $\alpha \neq \beta$.

Set

$$
\tilde{W}(x ; \lambda):=\hat{W}(x ; \lambda) e^{-\eta(x ; \lambda)} .
$$

By Lemma 4.4 this is local and as

$$
\tilde{W}(x ; \lambda)=1+O\left(\lambda^{-1}\right)
$$

it is an invertible matrix. Using these facts, the rest of the proof is the same as the arguments of [Ch1, F1, Sa and Fu-T]. 
Equation (4.8) implies

$$
\partial_{x_{n}^{(\alpha)}} \hat{W}(x ; \partial)=-\left(L^{n} U_{\alpha}\right)_{-}(x ; \partial) \hat{W}(x ; \partial),
$$

where $P_{-}:=P-P_{+}$. Using the Leibniz rule for micro-differential operators, we can expand the total symbol of (4.13) as follows:

$$
\partial_{x_{n}^{(\alpha)}} \hat{W}(x ; \lambda)=\sum_{j=1}^{\infty} \sum_{r=0}^{\infty}\left(\begin{array}{c}
-j \\
r
\end{array}\right) v_{\alpha, j}^{(n)}(x)\left(\partial^{r} \hat{W}(x ; \lambda)\right)^{\lambda-j-r} .
$$

Here local functions $v_{\alpha, j}$ 's are defined by $-\left(L^{n} U_{\alpha}\right)_{-}=\sum_{j=1}^{\infty} v_{\alpha, j}^{(n)} \lambda^{-j}$. Substituting (4.12) into (4.14), we obtain

$$
\begin{aligned}
& \partial_{x_{n}^{(\alpha)} \eta}(x ; \lambda) \\
& \quad=\tilde{W}^{-1}\left\{-\partial_{x_{n}^{(\alpha)}} \tilde{W}+\sum_{j=1}^{\infty} \sum_{r=0}^{\infty} \sum_{k=0}^{r}\left(\begin{array}{c}
-j \\
r
\end{array}\right)\left(\begin{array}{l}
r \\
k
\end{array}\right) v_{\alpha, j}^{(n)}(x) \partial^{k} \tilde{W}(x ; \lambda) \partial^{r-k} e^{\eta(x ; \lambda)} e^{-\eta(x ; \lambda) \lambda-j-r}\right\} .
\end{aligned}
$$

Note that $\left(\partial^{k} e^{\eta}\right) e^{-\eta}$ is a differential polynomial of $\partial \eta$ and not of $\eta$ itself. Therefore, if $\partial \eta$ is local, (4.15) proves the theorem, for the rest of the right-hand side of (4.15) is local as already mentioned.

Now we prove that $\partial \eta$ is local. Summing up (4.15) $n=1$ for all $\alpha$, we get $\partial \eta(x ; \lambda)$

$$
=\tilde{W}^{-1}\left\{-\partial \tilde{W}+\sum_{j=1}^{\infty} \sum_{r=0}^{\infty} \sum_{k=0}^{r}\left(\begin{array}{c}
-j \\
r
\end{array}\right)\left(\begin{array}{l}
r \\
k
\end{array}\right) v_{j}(x) \partial^{k} \tilde{W}(x ; \lambda) \partial^{r-k} e^{\eta(x ; \lambda)} e^{-\eta(x ; \lambda)} \lambda^{-j-r}\right\},
$$

where $v_{j}=v_{1, j}^{(1)}+\cdots+v_{r, j}^{(1)}$. It is an easy induction to show the locality of $\partial H_{\alpha, j}$ (and hence the locality of $\partial \eta$ ), comparing the coefficients of $\lambda^{-j}$ 's in (4.16).

Equation (4.9) yields

$$
\begin{aligned}
& \eta_{\alpha}(x ; \lambda)=\log \tau\left(x-\varepsilon_{\alpha}(\lambda)\right)-\log \tau(x), \\
& H_{\alpha, j}(x)=p_{j}\left(-\widetilde{\partial}_{x}^{(\alpha)}\right) \log \tau(x) .
\end{aligned}
$$

The conserved quantity obtained above is, by this formula,

$$
\oint \partial_{z} p_{j}\left(-\tilde{\partial}_{x}^{(\alpha)}\right) \log \tau(x) d z \text {. }
$$

In particular, when $r=2$ and $z=\frac{1}{2}\left(x_{1}^{(1)}+x_{1}^{(2)}\right)$, and provided that the solution considered is that of the TL hierarchy (cf. Theorem 4.1), then this is nothing but the Hamiltonian obtained in Sect. 3, Theorem 3.16. These conserved quantities and the coincidence of them play an important role in 2 dimensional field theory (cf. $[\mathrm{Fu}-\mathrm{T}]$ ).

Finally we make some remarks on this point.

Remark 4.5. Applying the method adopted in Sect. 4 to the TL hierarchy seems difficult, because the KP hierarchy is a system of differential equations while the TL hierarchy is a differential-difference system. Exactly speaking, the last argument in the proof of Theorem 4.3 does not work for the TL hierarchy. 
Remark 4.6. Conversely, applying the machinery in Sect. 3.2 to the KP hierarchy is, at least for the present, impossible, since the fundamental Poisson relation for the KP equation (or some reduced system of the KP hierarchy) has not yet been found.

So, it remains still mysterious that the conserved quantities of 2 component KP hierarchy and those of the TL hierarchy obtained through these methods coincide. Satisfactory explanation should be found in future studies.

Acknowledgements. The present author expresses his great thanks to Professors H. Komatsu, D. Olive, M. Jimbo, Kimio Ueno, and Doctors M. Noumi, H. Yamada, K. Takasaki, K. Ikeda, M. Fukuma, H. Ochiai for their hearty encouragement, advice and discussions.

\section{References}

[B-D] Belavin, A. A., Drinfel'd, V. G.: Solutions of the classical Yang-Baxter equation for simple algebras. Funks. Anal. i. ego Pril. 16, 1-29 (1982). In Russian, Funct. Anal. Appl. 16, 159-180 (1983)

[Ch1] Cherednik, I. V.: Differential equations for the Baker-Akhiezer functions of algebraic curves. Funks. Anal. i ego Pril. 12, 45-54 (1978). In Russian, Funct. Anal. Appl. 12, 195-203 (1978)

[Ch2] Cherednik, I. V.: Definition of $\tau$ functions for generalized affine Lie algebras. Funks. Anal. i ego Pril. 17, 93-95 (1983). In Russian, Funct. Anal. Appl. 17, 243-245 (1983)

[Fa] Faddeev, L. D.: Integrable models in $(1+1)$-dimensional quantum field theory. In: Développements récents en théorie des champs et mécanique statistique. Les Houches Summer School Session XXXIX, pp. 561-608. Amsterdam: North Holland 1984

[Fa-T] Faddeev, L. D., Takhtajan, L. A.: Hamiltonian methods in the theory of solitons. Moscow: Nauka 1986. In: Russian; in English. Berlin, Heidelberg, New York: Springer 1987

[F1] Flaschka, H.: Construction of conservation laws for Lax equations: Comments on a paper by G. Wilson. Quart. J. Math. Oxford, 34, 61-65 (1983)

[Fu-T] Fukuma, M., Takebe, T.: The Toda lattice hierarchy and deformations of conformal field theories. Univ. of Tokyo preprint, UT-547 (1989)

[G-Ch] Gel'fand, I. M., Cherednik, I. V.: The abstract Hamiltonian formalism for the classical Yang-Baxter bundles. Usp. Mat. Nauk 38, 3-21 (1983). In Russian, Russ. Math. Surv. 38, $1-22(1983)$

[G-D] Gel'fand, I. M., Dorfman, I. Ya.: Hamiltonian operators and related algebraic structures, Funkts. Anal. i ego Pril. 13, 13-30 (1979). In Russian, Funct. Anal. Appl. 13, 248-262 (1979)

[Ka] Kac, V. G.: Infinite dimensional Lie algebras, 2nd ed. Cambridge: Cambridge University Press 1985

[K-R] Kac, V. G., Raina, A. K.: Bombay Lectures on highest weight representations of infinite dimensional Lie algebras. Adv. Ser. Math. Phys. Vol. 2. Singapore: World Scientific 1987

[K-W] Kac, V. G., Wakimoto, M.: Exceptional hierarchies of soliton equations. Proc. of Symposia in Pure Mathematics 49, 191-237 (1989)

[Ko] Kotera, T.: Toda Lattice and Lie Algebra, Sophia Kokyuroku in Math Vol. 23. Lie algebras and Differential Equations, 91-100 (1986), in Japanese

[M-O-P] Mikhailov, A. V., Olshanetsky, M. A., Perelomov, A. M.: Two-Dimensional Generalized Toda lattice. Commun. Math. Phys. 79, 473-488 (1981)

[O-T1] Olive, D., Turok, N.: Algebraic structure of Toda systems. Nucl. Phys. B220 [FS8], 491-507 (1983)

[O-T2] Olive, D., Turok, N.: Local conserved densities and zero-curvature conditions for Toda lattice field theories. Nucl. Phys. B257 [FS14], 277-301 (1985)

[O-T3] Olive, D., Turok, N.: The Toda lattice field theory hierarchies and zero-curvature conditions in Kac-Moody algebras. Nucl. Phys. B265 [FS15], 469-484 (1986)

[R] Reiman, A. G.: A unified Hamiltonian system on polynomial bundles, and the structure of stationary problems. Zap. Nauchn. Semi. LOMI 131, Problems of quantum field theory 
and statistical physics IV, 118-127 (1983). In Russian, J. Sov. Math. 30, 2319-2326 (1985)

[R-S] Reyman, A. G., Semenov-Tian-Shansky, M. A.: Hamiltonian structure of equations of Kadomtsev-Petviashvili type. Zap. Nauchn. Semi. LOMI 133, Differential Geometry, Lie Groups and Mechanics VI, 212-227 (1984). In Russian, J. Sov. Math. 31, 3399-3410 (1985)

[Sa] Sato, M.: Lectures delivered at the Univ. of Tokyo., in Japanese

[Sk] Sklyanin, E. K.: Quantum version of the method of inverse scattering problem. Zap. Nauch. Semi. LOMI 95, 55-128 (1980), in Russian; J. Sov. Math. 19, 1546-1596 (1982)

[T] Takasaki, K.: On the Toda lattice and D modules, RIMS Kokyuroku 694, 1-19 (1989), in Japanese

[U-T] Ueno, K., Takasaki, K.: Toda lattice hierarchy. In: Group representation and systems of differential equations. Adv. Stud. Pure Math. Vol. 4, pp. 1-95. Tokyo: Kinokuniya 1984

[W] Wilson, G.: On two constructions of conservation laws for Lax equations. Quart. J. Math. Oxford, 32, 491-512 (1981)

Communicated by $\mathrm{H}$. Araki

Received September 20, 1989 